\title{
A Kinetic Theory of Trapped Electron Driven Drift Wave Turbulence in a Sheared Magnetic Field
}

\author{
F. Y. Gang ${ }^{a)}$ \\ Institute for Fusion Studies \\ University of Texas at Austin \\ Austin, TX 78712
P. H. Diamond and M. N. Rosenbluth
Department of Physics B-019
University of California, San Diego
La Jolla, CA 92093
and
General Atomics, Inc
San Diego, CA 92138

$\mathrm{DOE} / \mathrm{ET} / 53088--456$

DE91 004302

\section{Abstract}

A kinetic theory of collisionless and dissipative trapped electron driven drift wave turbulence in a sheared magnetic field is presented. Weak turbulence theory is employed to calculate the nonlinear electron and ion responses and to derive a wave hinetic equation that determines the nonlinear evolution of trapped electron mode turbulence. Saturated fluctuation spectrum is calculated using the condition of nonlinear saturation. The turbulent transport coefficients $\left(D, \chi_{i}, \chi_{e}\right)$ are in turn calculated using saturated fluctuation spectrum. Due to the disparity in the three different radial scale lengths of the slab-like eigenmode: $\Delta$ (trapped electron layer width), $x_{t}$ (turning point width) and $x_{i}$ (Landau damping point), $\Delta<x_{\ell}<x_{i}$, we find that ion Compton scattering rather than trapped electron Compton scattering is the dominant nonlinear saturation mechanism. Ion Compton scattering transfers wave energy from short to long wavelengths where the wave energy is shear damped. As a consequence, a saturated fluctuation spectrum $|\phi|^{2}\left(k_{\theta}\right) \sim k_{\theta}^{-\alpha}(\alpha=2$ and 3 for the dissipative and collisionless regime, respectively) occurs for $k_{\theta} \rho_{s}<1$ and is heavily damped for $k_{\theta} \rho,>1$. The predicted fluctuation level and transport coefficients are

a) present address: Department of Physics B-019, University of California, San Diego, La Jolla, Ca 92093

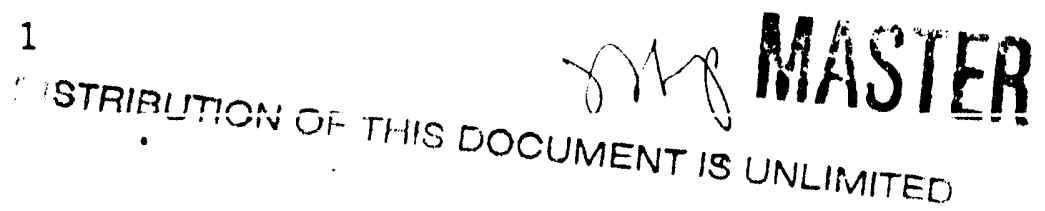


well below the 'mixing length' estimate. This is due to the contribution of radial wavenumbers $x_{t}^{-1}<k_{r} \leq \rho_{i}^{-1}$ to the nonlinear couplings, the effect of radial localization of trapped electron response to a layer of width $\Delta$, and the weak turbulence factor $\left\langle\frac{\gamma_{a}^{\prime}}{\omega_{\vec{k}}}\right\rangle_{\bar{k}}<1$, which enters the saturation level.

\section{DISCLAIMER}

This report was prepared as an account of work sponsored by an agency of the United States Neither the United States Government nor any agency thereof, nor any of their Government. Neither the United States Government nor ans implied, or assumes any legal liability or responsiemployees, makes any warranty, express or implied, or assumes any legal liability or reduct, or bility for the accuracy, completeness, or usefulness of any information, apparatus, product, process disclosed, or represents that its use would not infringe privately owned rights. Refade name, trademark, ence herein to any specific commercial product, process, or service by trade name, its endorsement, recommanufacturer, of otherwise does not necessarily constitute or imply its endores mendation, or favoring by the United States hovern do not necessarily state or reflect those of the and opinions of authors expressed herein do 


\section{Introduction}

Trapped electron driven drift wave turbulence, both dissipative and collisionless, is considered to be an important agent for anomalous transport in tokamaks. While the linear theory of trapped electron instabilities has been extensively investigated, ${ }^{1-3}$ the nonlinear dynamics of trapped electron drift wave turbulence remaines rather poorly understood. Indeed, with relatively few exceptions, ${ }^{4-7}$ most previous studies of trapped electron driven drift wave turbulence have forsalen realistic geometries in favor of shearless slais models, ${ }^{8}$ adopted the fluid ion approximation ab initio, ${ }^{9,10}$ and have ignored the nonlinear dynamics of trapped electrons by a priori invocation of the " $i \delta$ " representation of the non-adiabatic electron response. ${ }^{3}$ The purpose of this investigation is to explore the theory of trapped electron drift wave turbulence, with special attention focused on the role of ion kinetic effects in the nonlinear dynamics and on the role of trapped electron nonlinearity. In order to elucidate the fundamental physics issues, we adopt the comparatively simple, albeit unrealistic, model of sheared slab geometry with magnetically trapped electrons. This model ignores the poloidal harmonic couplings induced by ion curvature drifts, and thus describes only the short wavelength, slab-like branch of trapped electron drift wave turbulence. The nonlinear theory of the long wavelength, toroidicity induced branch will be discussed in a future publication. It should be noted however, that despite the absence of shear damping of the toroidicity induced mode, it is not clear whether the slab or toroidal branch is more important agent for anomalous transport, since conventional mixing length estimates suggest that the thermal transport coefficient (in the dissipative regime, for example) for the slab branch is $\chi_{\text {slab }} \simeq \sqrt{\varepsilon}\left\langle k_{\theta} \rho_{s}\right\rangle_{\text {rms }} \frac{\rho_{s}^{2} c_{1}^{2}}{\nu_{e s} L_{n}^{2}} \sqrt{\frac{L_{1}}{L_{n}}}$, while for the toroidal branch is $\chi_{\text {tor }} \simeq \sqrt{\epsilon} \frac{\rho_{1}^{2} c_{1}^{2}}{\nu_{e} s L_{n}^{2}}$.

A key feature of trapped electron driven drift wave turbulence in a sheared slab is the disparity between the three radial scales $\Delta, x_{t}$, and $x_{i}$ which parametrize a trapped electron eigenmode. Specifically, the three scales are: i.) the separation of adjacent mode rational surfaces for fixed toroidal mode number $n, \Delta=\frac{1}{k_{0} \dot{s}}$. $\Delta$ is also the trapped 
electron layer width, which demarks the region in which the trapped electron response is significant. $\Delta$ arises as a consequence of the fact that trapped electrons tend to bounce along magnetic field lines (i.e. $h_{n}^{e} \sim e^{-i n q \theta} \tilde{h}_{n}^{e}$, where $h_{n}^{e}$ is the nonadiabatic part of trapped electron distribution perturbation, $q$ is the safety factor) while drift waves tend to localize at mode rational surfaces $\left(\phi_{n} \sim \sum_{m} e^{i m \theta} \phi_{n}^{m}\right)$. ii.) the turning point width $x_{t}=\sqrt{\frac{L_{1}}{L_{n}}} \rho_{9}$, where $L_{s}, L_{n}$, and $\rho_{s}$ are the magnetic shear length, equilibrium density scale length, and ion larmor radius computed using the electron temperature. $x_{t}$ demarks the extent of the region over which the Pearlstein-Berk ${ }^{11}$ outgoing wave eigenmode is non-oscillatory, i.e. for $\phi_{\vec{k}}=\exp \left\{-i \mu \frac{x^{2}}{2}\right\}$ with $\mu=x_{t}^{-2}$, and is defined by the asymptotic balance of the ion polarization drift $\rho_{s}^{2} \frac{\partial^{2} \phi_{\vec{k}}}{\partial x^{2}}$ with the ion acoustic response $\frac{k_{\|}^{2} c_{1}^{2}}{\omega^{2}} \phi_{\vec{k}}$, where $k_{\|}$is the parallel wavenumber and $c_{s}$ is the ion acoustic speed. More generally, $x_{t}$ is the scale at which the drift wave changes character from a quasi-two dimensional "convective cell" to a radially propagating outgoing wave. Thus, nonadiabatic electron effects contributes only for $x<x_{t}$, and $x_{t}$ is ggenerally assumed to be the "mixing length" for slab-like electron drift wave turbulence (i.e. the effective mode width). iii.) the ion Landau damping point $x_{i}=\left(\frac{L_{L}}{L_{n}}\right) \sqrt{\frac{T_{e}}{T_{i}}} \rho_{s}$, where $T_{e}$ and $T_{i}$ are the electron and ion temperature, respectively. $x_{i}$ defines the scale at which the outgoing wave is damped by ion Landau resonance (i.e. when $\left.\omega=k_{\|} v_{i}\right)$. Thus, $x_{i}$ charaterizes the radial width of the spectrum $\left|\phi_{\vec{k}}\right|^{2}$ of drift wave turbulence, in that if $\phi_{\bar{k}}=\exp \left(-i \mu \frac{x^{2}}{2}\right) f\left(\frac{x}{x_{i}}\right)$, with $x_{i}^{2} \mu \gg 1$, the fast variation cancels u.pon calculating $\left|\phi_{\vec{k}}\right|^{2}$ (i.e. $\left|\phi_{\vec{k}}\right|^{2}=f^{2}\left(\frac{x}{x_{i}}\right)$ ). It is interesting to note that for $x_{t}<x<x_{i}$, the drift wave develops large radial wave-numbers $k_{r}=-\mu x=-\frac{x}{x_{t}^{2}} \leq \rho_{i}^{-1}$. For most $k_{\theta} \rho_{\mathrm{s}}$ of interest, $\Delta<x_{t} \ll x_{i}$.

Several crucial aspects of the theory of trapped electron drift wave turbulence are revealed by consideration of the scales $\Delta, x_{t}, x_{i}$ and their disparity. First, the fact that the spectrum width is given by $x_{i}$ ensures that the virtual, driven modes (i.e. those driven by the beat interaction $\left(\omega+\omega^{\prime}, \vec{k}+\vec{k}^{\prime}\right)$ of a test wave $(\omega, \vec{k})$ and background mode $\left.\left(\omega^{\prime}, \vec{k}^{\prime}\right)\right)$ are resonant, on account of the natural width of the ballistic frequency 
$k_{i \|}^{\prime} v_{\|}$, i.e. for resonance, $\omega+\omega^{\prime} \leq \Delta\left(k_{\|}^{\prime} v_{\|}\right)$, but $\Delta\left(k_{\|}^{\prime} v_{\|}\right) \sim \omega^{\prime}$, since $\Delta\left(k_{\|}^{\prime}\right)=\frac{k_{g}^{\prime}}{L_{i}} x_{i}^{\prime}$. This observation indicates that while the fluid ion approximation may be valid for test wave dynamics, where $x<x_{t}$ is of greatest interest, it is a manifestly invalid description of the nonlinear interaction dynamics, since it ignores the beat-wave resonance $\omega^{\prime \prime} \sim k_{\|}^{\prime \prime} v_{i}$ and thus fails to represent nonlinear ion Landau damping and Compton scattering. Moreover, it is interesting to note that while ion fluid mode coupling among strongly dispersive drift waves requires large amplitude dependent turbulent broadening of the three-wave frequency mismatch $\omega_{M M} \sim \omega \sim \Delta$, , the kinetic beat wave resonance is Doppler broadened at infinitesimal amplitudes by the spatial extent of the background mode spectrum $x_{i}$, i.e. $\Delta\left(k_{\|} v_{i}\right) \sim k_{\theta} / L_{s} x_{i} v_{i} \sim \omega$. As $\omega \geq \Delta \omega$, the Doppler broadening is the more significant one. These two rbservations suggest that nonlinear ion Landau damping and Compton scattering, as described by kinetic weak turbulence theory (since $\gamma<\omega_{r}$ ), are importint (and likely dominant!) processes in the nonlinear dynamics of slab-like irapped electron modes. Finally, it is also worthwhile to note that since $x^{\prime}$ extends to $x_{i}^{\prime}, k_{r}^{\prime}$ can approch $\rho_{i}^{-1}$. Thus, radial wavenumbers $k_{r} \leq \rho_{i}^{-1}$ contribute to the 'coupling coefficient' $\vec{k} \cdot \vec{k}^{\prime} \times \vec{z}$. Since, $k_{r}^{\prime}>x_{t}^{-1}$, saturation at levels $\frac{\tilde{n}}{n_{0}}$ below the conventionally quoted 'mixing length estimated' of $\frac{\tilde{n}}{n_{0}} \sim \frac{x_{0}}{L_{n}}$ appears likely!

A second crucial feature of trapped electron modes is the disparity between the width of the electon layer $\Delta$ on one hand and the mode width $x_{t}$ and spectral width $x_{i}$ (i.e. $\left.\Delta<x_{t}<x_{i}\right)$, on the other. This disparity underlines the dominance of ion nonlinearity over electron nonlinearity, since electron interaction is limited to $|x|<\Delta$, while the region of nonlinear ion-mediated interaction extends to $x_{i}$. Indeed, the effect of localization of trapped electron effects to a layer of width $\Delta$ has already been manifested in the linear theory ${ }^{2}$ of trapped electron instabilities, where calculated linear growth rates are smaller than their local theory analogy by a factor $\frac{\Delta}{x_{t}} \ln \frac{x_{t}}{\Delta}<1$, i.e. the ratio of the electron layer width to the mode width. Of course, in the case of toroidicity induced drift modes ${ }^{12}$ for which the mode and the spectral widths are comparable to $\Delta$, the nonlinear dynamics 
of the collisionless trapped electrons are not subdominant, since the electron layer width correction factor approaches unity.

In this paper, a kinetic theory of collisionless and dissipative trapped electron drift wave turbulence is presented. A slab-like model (i.e. magnetically trapped electrons, with ion curvature effects neglected) is used throughout. Nonlinear trapped electron and ion interaction mechanisms are treated on an equal footing. A nonlinear bounce-kinetic equation is used to calculate the nonlinear (collisionless) trapped electron response perturbatively. While nonlinear trapped electron-wave interaction tends to transfer energy from long to short wavelengths, it is subdominant to the nonlinear ion-wave interaction process, since $\Delta \ll x_{t}, x_{i}$. The nonlinear ion response is also calculated perturbatively, following the standard procedures of weak turbulence theory ${ }^{13}$. The Doppler broadening of the beat wave resonance induced by the large ion spectral width $\left(x_{i}\right)$ indicates that ion Compton scattering is a relevant and robust nonlinear interaction process. Ion Compton scattering results in spectral transfer to long wavelengths, primarily by local interaction. A power law spectrum $|\phi|^{2}\left(k_{\theta}\right) \sim k_{\theta}^{-\alpha}(\alpha=2$ and 3 for dissipative and collisionless regimes, respectively) results, with a low- $k_{\theta}$ cutoff determined by the balance of linear growth with shear damping. Fluctuation levels are lower than naive mixing length prediction $\frac{\tilde{n}}{n_{0}} \sim \frac{x_{1}}{L_{n}}$. The calculated fluctuation spectrum is used to calculate the electron and ion thermal diffusivities, and the particle flux.

At this point it is appropriate to comment on the relation of this investigation to previous works of similar bent. Refs.(4) and (5) treat only nonlinear ion dynamics for slab-like and toroidal drift modes, respectively, and neglect electron nonlinearity, ab initio. Ref.(6) discusses nonlinear trapped electron dynamics in the context of a purely local model, which therefore ignores the disparity between $\Delta, x_{t}$ and $x_{i}$. Thus, the conclusions reached in that work are not universal. With regards to ion dynamics, some of the conclusions reached here concerning the relative importance of local and nonlocal transfer processes and the structure of the predicted fluctuation spectrum differ with those presented in Ref.(4). This 
is, in part, due to exceedingly large value of $T_{e} / T_{i}$ tacitly assumed in that investigation. Finally, Ref.(7) is exclusively focused on the effect of mode coupling mediated by resonant trapped electrons (i.e. trapped electron clumps) and on the resultant broadening of the frequency linewidth.

The remainder of this paper is organized as follows. In Sec.II, we discuss the model equations. In Sec.III, the linear theory of the trapped electron mode is reviewed. In Sec.IV, weak turbulence theory is employed to calculate the nonlinear ion response and the nonlinear trapped electron response. In Sec.V, we derive a wave kinetic equation which describes the nonlinear evolution of trapped electron mode turbulence. In Sec.VI, we discuss the various nonlinear wave-particle interaction mechanisms and calculate the associated nonlinear transfer rate. The complications of slab-like eigenmode structure for the nonlinear wave particle interaction are emphasized. In Sec.VII, the saturated fluctuation spectrum and fluctuation level are calculated. In Sec.VIII, the saturated fluctuation spectrum obtained in the previous section is used to calculate the ion and electron thermal transport coefficients, and the particle transport coefficient. The implications of these results to the tolamak transport are then discussed. In Sec.IX, the summary and conclusion are presented.

\section{II: Basic Model}

In this section, the basic model of trapped electron driven drift wave turbulence is discussed. We consider toroidal geometry with circular concentric magnetic surfaces, parametrized by the usual coordinates $(r, \theta, \xi)$ corresponding to the minor radius, the poloidal angle and the toroidal angle, respectively. In this coordinate system, the equilibrium magnetic field can be written as $\vec{B}=B\left(\vec{e}_{\xi}+\frac{\epsilon}{q} \vec{e}_{\theta}\right)$, where $\epsilon=\frac{r}{R_{0}}$ is the inverse aspect ratio, $R_{0}$ is the major radius from the magnetic axis, $B=B_{0}(1-\epsilon \cos \theta)$ is the magnitude of the magnetic field, $q$ is the safty factor, and $\vec{e}_{\theta}, \vec{e}_{\xi}, \vec{e}_{r}$ are the unit vectors in the poloidal, toroidal and radial directions, respectively. The equilibrium distribution 
functions are assumed to be local Maxwellians, with density $\mathrm{N}$ and temperature $T_{j}$, i.e.

$$
F_{j}=N\left(\frac{M_{j}}{2 \pi T_{j}}\right)^{\frac{3}{2}} \exp \left\{-\frac{M_{j} v^{2}}{2 T_{j}}\right\}, j=i, e
$$

where $M_{j}$ is the mass, $\mathrm{v}$ is the velocity, and $\mathrm{j}$ denotes the species.

For the sake of simplicity, we neglect both ion and electron temperature gradient in this analysis. We also assume that the electron temperature $T_{e}$ is higher than the ion temperature $T_{i}$.

For the perturbed ion distribution function $f^{i}$ and electrostatic potential perturbation $\phi$, we can Fourier analyze in $\theta$ and $\xi$ and time $t$,

$$
\left[f^{i}, \phi\right]=\sum_{i}\left[f_{!}^{i}, \phi_{!}\right] \exp \{i(m \theta-n \xi-\omega t)\}
$$

where $l \equiv(m, n)$, and $m, n$ are the poloidal and toroidal mode numbers, respectively. The ion's dynamics is described by the nonlinear gyrokinetic equation, ${ }^{14}$

$$
-i\left(\omega-k_{\|} v_{\|}\right) h_{\omega}^{i}+i \frac{e}{T_{i}} F_{i}\left(\omega-\omega_{i}^{*}\right) J_{0}\left(\frac{k_{\perp} v_{\perp}}{\Omega_{i}}\right) \phi_{\downarrow}=N_{!}
$$

with the nonlinear mode coupling term $N_{l}$ given by

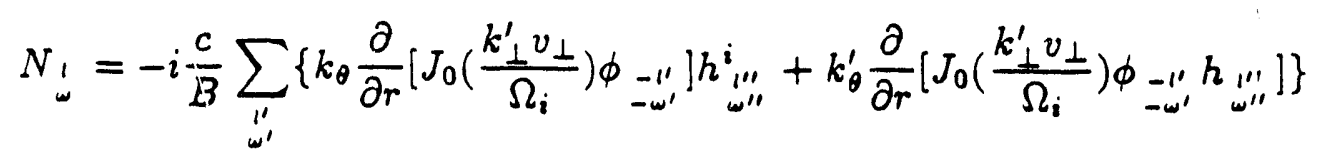

where $l^{\prime \prime}=l+l^{\prime}, \omega^{\prime \prime}=\omega+\omega^{\prime}$. In the above equations, $h^{i}$, is the nonadiabatic part of ion distribution perturbation in the guiding center system, and is related to $f_{i}^{i}$ through $f_{i}^{i}=-\frac{e}{T_{i}} F_{i} \phi_{1}+h_{i}^{i} e^{i \bar{i}_{\|} \cdot \frac{\vec{i}_{1} \times \sigma_{1}}{n_{i}}}, \vec{e}_{\| \mid}$is the unit vector in the direction of the local magnetic field, $\Omega_{i}$ is the ion gyrofrequency, $J_{0}$ is the zeroth order Bessel function, $k_{\|}=\frac{1}{q R}(m-n q)$ is the parallel wavenumber, $\omega_{i}^{*}=-\frac{c T_{i}}{e B} \frac{k_{\theta}}{L_{n}}$ is the ion diamagnetic drift frequency, $k_{\theta}=\frac{m}{r}$ is the poloidal wavenumber, $k_{\perp}$ is the perpendicular wavenumber, $\vec{k}_{\perp}=k_{\theta} \vec{e}_{\theta}+k_{r} \vec{e}_{r}$, and $k_{r}=-i \frac{\partial}{\partial r}$. The ion density perturbation is given by

$$
\delta n_{i}^{i}=-\frac{e}{T_{i}} N \dot{\phi}_{!}+\int d^{3} \vec{v} J_{0}\left(\frac{k_{\perp} v_{\perp}}{\Omega_{i}}\right) h_{i}^{i}
$$


For trapped electrons, we assurne $\nu_{e f f}, \omega_{D e}, \omega<\omega_{b e}$, where $\nu_{e f f}=\frac{\nu_{e i}}{\epsilon}$ is the effective collision frequency, and $\omega_{D e}, \omega_{b e}$ are the electron magnetic precession and bounce frequencies, respectively. In the collisionless regime, we have $\omega>\omega_{D e}>\nu_{e f f}$, so that the mode is destabilized by the magnetic precession resonance of high energy trapped electrons. In the dissipative regime (strong collisional regime), we have $\nu_{e f f}>\omega>\omega_{D e}$, so that the mode is destabilized by the collisional detrapping effect. In the intermediate collisional regime $\left(\omega>\nu_{e f f}>\omega_{D e}\right)$, the growth rate of the mode has such a favorable temperature dependence $\left(\gamma_{e}^{l} \propto T_{e}^{-2}\right)$ that it seems quite likely that the turbulence will eventually evolve into the collisionless regime. Therefore, in this discussion, we focus primarily on the collisionless and dissipative regime.

Since trapped electrons can induce poloidal couplings, the electron perturbed distribution function $f^{e}$ and the electrostatic potential perturbation $\phi$ are Fourier analyzed only in $\xi$ and $t$,

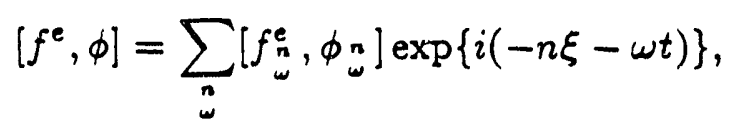

The trapped electron dynamics is described by a nonlinear bounce-kinetic equation, ${ }^{15}$

$$
-i\left(\omega-\omega_{d e}+i \tilde{\nu}_{e f f}\right) \hat{h}_{n}^{e}-i \frac{e}{T_{e}} F_{e}\left(\omega-\omega_{e}^{*}\right)\left\langle e^{-i n q \theta} \psi_{\omega}^{\prime}\right\rangle_{b}=N_{n}^{e}
$$

where the nonlinear mode coupling term is

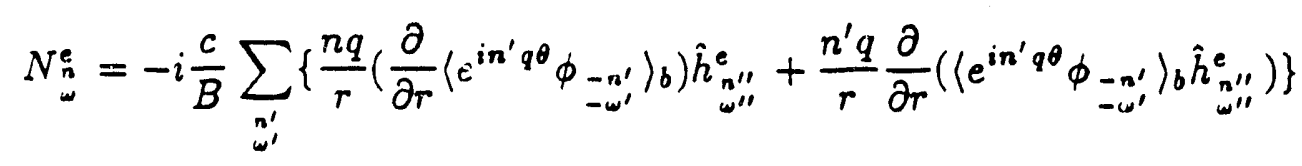

In the above equations, $\hat{h}_{n}^{e}$ is the nonadiabatic part of the trapped elertrnn distribution perturbation, and is related to $f_{n}^{e}$ through ${\underset{w}{e}}_{w}^{e}=\frac{e}{T_{0}} F_{e} \phi_{n}+\hat{h}_{n}^{e} e^{i n q \theta}, \omega_{d e}=\omega_{D e} \frac{v^{2}}{v_{e}^{2}}, \omega_{D e}=$ $\frac{L_{n}}{R} \omega_{e}^{*}, \omega_{e}^{*}=\frac{c T_{f}}{e B} \frac{k_{g}}{L_{n}}, \tilde{\nu}_{e f f}=\nu_{e f f}\left(\frac{v_{c}}{v}\right)^{3}$, and $\langle\cdots\rangle_{b} \equiv\left[\oint \frac{d \theta}{v_{\|}} \cdots\right] /\left[\oint \frac{d \theta}{v_{\|}}\right]$denotes a bounce average. The untrapped electron is treated as adiabatic. The electron density perturbation is then given by

$$
\delta n_{\omega}^{e}=\frac{e}{T_{e}} N \underset{\omega}{\dot{q}}+\int_{t} d^{3} \vec{v} h_{\omega}^{e} e^{i n q \theta}
$$


where the velocity integration is over the trapped electron population only. The velocity space variables in Eqs.(4) and (7) are the velocity $v$, the magnetic moment $\mu=\frac{v_{1}^{2}}{2 B}$ and the gyrophase angle $\varphi$. Finally, by imposing the quasineutrality condition $\delta n_{\substack{n \\ n}}^{i}=\delta n_{\substack{m \\ n}}^{e}$, Eqs. (3)-(4) and (6)-(7) are closed.

\section{Review of linear theory}

In this section, the linear theory of trapped electron mode is reviewed with attention focused on those properties that are important for an understanding of the nonlinear evolution of the mode. Included are the mode frequency, the growth rate, and the mode structure. For a detailed discussion, the reader is refered to Ref.(2). Linearizing Eq.(3) and Eq.(6), we obtain the linear responses for both ions and trapped electons,

$$
\begin{aligned}
& h_{l}^{i}=\frac{e}{T_{i}} F_{i} \frac{\omega-\omega_{i}^{*}}{\omega-k_{\|} v_{\|}} J_{0}\left(\frac{k_{\perp} v_{\perp}}{\Omega_{i}}\right) \phi_{\omega} \\
& \hat{h}_{n}^{e}=-\frac{e}{T_{e}} F_{e} \frac{\omega-\omega_{e}^{*}}{\omega-\omega_{d e}+i \tilde{\nu}_{e f f}}\left\langle e^{-i n q \theta} \phi_{n}\right\rangle_{b}
\end{aligned}
$$

It is important to note that trapped electrons respond to $\left\langle e^{-i n q \theta} \phi_{n}\right\rangle_{b}$, the bounce average of the projection of the potential fluctuation along the magnetic field line, rather than the potential fluctuation $\phi_{n}$ itself. In the presence of magnetic shear, the pitch of the magnetic field line is different from that of the mode, i.e., $n q(r) \neq m$, except at the mode rational surface. Away from the mode rational surface, due to its fast oscillation, the bounce average of $e^{-i n q \theta} \phi_{n}$ tends to vanish. Therefore the trapped electron's response is radially localized with a spatial scale length much smaller than the mode width. Physically, this is equivalent to saying that the trapped electrons can only feel the potential fluctuation near mode rational surfaces, where the difference between the pitch of the magnetic field line and that of the mode is negligible. As a consequence of this radial localization effect, the growth rate of the mode is considerably reduced. The fact that trapped electrons respond to the magnetic field line rather than the mode rational surface also introduces poloidal couplings among modes which center at different mode rational surfaces. However, as pointed out by Catto and Tsang in Ref.(2), this poloidal coupling effect is rather weak. 
Now, we can use Eq.(4) and Eq.(7) to calculate the ion and the electron density response. For ions the calculation is straight forward. To perform the velocity integration over the trapped electron population, we note that $d^{3} \vec{v} \simeq 4 \pi\left(\frac{\epsilon}{2}\right)^{\frac{1}{2}} v^{2} d v d \kappa^{2}\left(\kappa^{2}-\sin ^{2} \frac{\theta}{2}\right)^{-\frac{1}{2}}$, where instead of using variables $v$ and $\mu$, we use $v$ and $\kappa, \kappa$ is the pitch angle variable, and is defined by $\kappa^{2}=\left[\frac{1}{2} v^{2}-\mu B_{0}(1-\epsilon)\right] / 2 \epsilon \mu B_{0}$. We then Fourier transiorin $\delta n_{n}^{e}$ in $\theta$, i.e., $\delta n_{\substack{n \\ n}}^{e}=\frac{1}{2 \pi} \int_{-\infty}^{\infty} d \theta e^{-i m \theta} \delta n_{n}^{e}$, and invert the order of the $\theta$ and $\kappa^{2}$ integrations by noting that $\int_{-\pi}^{\pi} d \theta \int_{\kappa_{0}}^{1} d \kappa^{2}=\int_{0}^{1} d \kappa^{2} \int_{-\theta_{0}}^{\theta_{0}} d \theta$, where $\kappa_{0}=\sin \frac{\theta_{0}}{2}$. The final results of the calculation are,

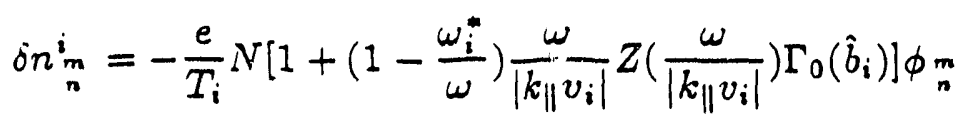

and

$$
\begin{aligned}
\delta n_{n}^{e} & =\frac{e}{T_{e}} N\left(\phi_{n}^{m}+\hat{\Upsilon}_{e}^{l}\left\langle e^{-i n q \theta} \phi_{n}\right\rangle_{b}\right) \\
\hat{\Upsilon}_{e}^{\prime}\left\langle e^{-i n q \theta} \phi_{n}\right\rangle_{b} & =\left(\frac{\epsilon}{2}\right)^{\frac{1}{2}}\left(1-\frac{\omega_{e}^{*}}{\omega}\right) g_{n} \int_{0}^{1} d \kappa^{2} \overline{e^{i(n q-m) \theta}}\left\langle e^{-i n q \theta} \phi_{n}\right\rangle_{b}
\end{aligned}
$$

In the above equations, $Z$ is the plasma dispersion function, $\Gamma_{0}$ is the zeroth order modified Bessel function, $b_{i}=\frac{1}{2} k_{\perp}^{2} \rho_{i}^{2}, \overline{e^{i(n q-m) \theta}}$, and $g_{n}$ are defined as

$$
\begin{aligned}
& \overline{e^{i(n q-m) \theta}}=\int_{-\theta_{0}}^{\theta_{0}} \frac{d \theta}{2 \pi} \frac{e^{i(n q-m) \theta}}{\sqrt{\kappa^{2}-\sin ^{2} \frac{\theta}{2}}} \\
& g_{n}=-\frac{2}{\sqrt{\pi}} \int_{t_{0}}^{\infty} \sqrt{t} d t \frac{e^{-t}}{1-\frac{\omega_{R} a}{\omega} t+i \frac{\nu_{0} l l}{\omega} t^{-\frac{3}{2}}}
\end{aligned}
$$

where $t_{0}=\left(\frac{q R \nu_{e}}{e^{\frac{3}{2}} v_{a}}\right)^{\frac{1}{2}} \ll 1$

Let's consider a reference mode rational surface located at $r \underset{n}{m}$ determined by $m=$ $n q\left(r_{n}^{m}\right)$, then $k_{\|}=\frac{k_{9}}{L_{0}} x$, where $x=r-r_{n}^{m}$ is the distance from mode rational surface, $L_{s}=\hat{s}^{-1} q R$ is the shear length, and $\hat{s}=-\frac{d \ln q}{d \ln r}$ is the shear parameter. We define the Landau resonance point $x_{i} \equiv \frac{\omega}{k_{\|}^{\prime} v_{i}}$ where $k_{\|}^{\prime}=\frac{k_{A}}{L_{\bullet}}$. Then, in the spatial region $x>x_{i}$, the mode is heavily Landau damped. Hence, the mode exists only in the region $x<x_{i}$. By making the fluid ion approximation $\omega \gg k_{\|} v_{\|}$, and noting that $b_{i}<1$ for $\frac{T_{i}}{T_{e}}<1$, Eq.(9) 
can be simplified to be,

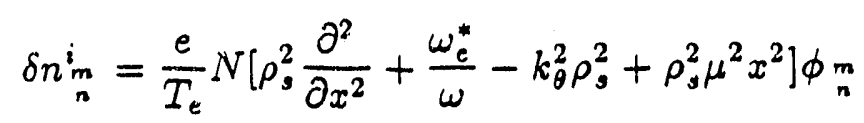

where $\rho_{\mathrm{g}}$ is the ion larmor radius computed using electron temperature, and $\rho_{s}^{2} \mu^{2}=\left(\frac{k_{y}^{\prime} c_{0}}{\omega}\right)^{2}$. By imposing the quasineutrality condition, we obtain the linear eigenmode equation,

$$
\left[\rho_{s}^{2} \frac{\partial^{2}}{\partial x^{2}}+\frac{\omega_{e}^{*}}{\omega}-k_{\theta}^{2} \rho_{s}^{2}-1+\rho_{s}^{2} \mu^{2} x^{2}\right] \phi_{n}^{m}=\hat{\Upsilon}_{e}^{\prime}\left\langle e^{-i n q \theta} \phi_{n}\right\rangle_{b}
$$

It is important to note that the above eigenmode equation has three distinct spatial scales, $\Delta, x_{t}$, and $x_{i}$ (see Fig.1). Here, $\Delta=\frac{1}{\left|k_{\theta}\right| \dot{j}}$ is the separation between two adjacent mode rational surfaces for fixed $n, x_{t}=|\mu|^{-\frac{1}{2}}$ is the turning point of the mode, and $x_{i}=\frac{\omega L_{l}}{k_{\theta} v_{i}}$ is the Landau resonance point. The trapped electron response (the right side of Eq.(13)) varies on the scale $\Delta$, the linear mode itself $\underset{n}{\phi_{n}}$ varies on the scale of $x_{t}$, and the spectrum $\left|\phi_{n}\right|^{2}$ varies on the scale $x_{i}$. For $k_{\theta} \rho_{s} \sim 1$, we observe that $\frac{\Delta}{x_{i}} \sim \sqrt{\frac{L_{n}}{L_{0}}}$, and $\frac{\Delta}{x_{i}} \sim \frac{L_{n}}{L_{0}} \sqrt{\frac{T_{i}}{T_{i}}}$. Each eigenmode, centered at a particular mode rational surface $r_{n}^{m}$, interacts with as many as $\frac{L_{2}}{L_{n}}(\gg 1)$ mode rational surfaces in it's neighborhood. In this case, the linear eigenmodes are said to be densely packed. A schematic picture of linear eigenmode structure with three spatial scale lengths $\Delta, x_{t}$, and $x_{i}$ is shown in Fig.1.

Equation (13) can be solved by treating the right side perturbatively. In lowest order, the solution is the Pearlstein-Berk outgoing wave function. For the most unstable mode ( i.e. least shear darnped), we have

$$
\phi_{n}^{m}=\hat{\phi}_{n} e^{-i \mu \frac{x^{2}}{2}}
$$

The outgoing wave boundary condition requires that $\mu$ should be an odd function of $k_{\theta}$, i.e.

$$
\mu=\frac{\omega_{e}^{*}}{|\omega|} \frac{L_{n}}{L_{s}} \frac{1}{\rho_{s}^{2}}
$$

The linear dispersion relation is determined by equation $\varepsilon\left(\vec{k}, \omega_{\vec{k}}\right)=0$, where the linear dielectric function is $\varepsilon(\vec{k}, \omega)=\frac{\omega_{\omega}^{*}}{\omega}-k_{\theta}^{2} \rho_{g}^{2}-1-i \mu \rho_{s}^{2}$. The linear frequency and shear 
damping rite are

$$
\begin{aligned}
\omega_{\vec{k}} & =\frac{\omega_{e}^{*}}{1+k_{\theta}^{2} \rho_{s}^{2}} \\
\gamma^{\text {sd }} & =-\frac{I_{n}}{L_{s}}\left|\omega_{\vec{k}}\right|
\end{aligned}
$$

We next obtain the linear growh rate $\mu_{e}^{l}$ due to the trapped electron excitation. From perturbation theory, we have

$$
\varepsilon(\vec{k}, \omega)=\frac{\int_{-\infty}^{\infty} d x \phi_{n} \hat{\Upsilon}_{e}^{\prime}\left\langle e^{-i n q \theta} \phi_{n}\right\rangle_{b}}{\int_{-\infty}^{\infty} d x \phi_{m}^{2}}
$$

where $\omega=\omega_{\vec{k}}+i \gamma^{\text {sd }}+i \gamma_{e}^{l}$. The detailed calculation of the spatial integrals in the above equation has been given in Ref.(2). Here, we only summarize some of the important results of the calculation. The spatial integral in the denominator of the above equation is easily evaluated as

$$
\int_{-\infty}^{\infty} d x \phi_{n}^{2}=\hat{\phi}_{n}^{2} \sqrt{\frac{T}{i \mu}}
$$

The spatial integral in the numerator, which involves the poloidal coupling effects, can be rewritten as

$$
\begin{aligned}
& \int_{-\infty}^{\infty} d x{\underset{n}{m}}_{n} \hat{\Upsilon}_{e}^{l}\left\langle e^{-i n q \theta} \phi_{n}\right\rangle_{b} \\
& =\left(\frac{\epsilon}{2}\right)^{\frac{1}{2}}\left(1-\frac{\omega_{e}^{*}}{\omega}\right) g_{n} \sum_{p=-\infty}^{\infty} \int_{0}^{1} d \kappa^{2} \int_{-\infty}^{\infty} d x \underset{n}{\phi_{n}^{m} \phi_{n}^{m+p}} \overline{e^{i(n q-m) \theta}}\left\langle e^{i(m+p-n q) \theta}\right\rangle_{b}
\end{aligned}
$$

As has been shown in Ref.(2), by beeping all $p$ terms in Eq.(20), the integral is only a factor of $\frac{\pi}{2}$ times that obtained by keeping the $p=0$ term only. Hence, we can approximate Eq. (20) as

$$
\begin{aligned}
& \int_{-\infty}^{\infty} d x \dot{\phi}_{n}^{n} \hat{\Upsilon}_{e}^{\prime}\left\langle e^{-i n q \theta} \phi_{n}\right\rangle_{b} \\
& =\frac{\pi}{2}\left(\frac{\epsilon}{2}\right)^{\frac{1}{2}}\left(1-\frac{\omega_{e}^{*}}{\omega}\right) g_{n} \int_{0}^{1} d \kappa^{2} \int_{-\infty}^{\infty} d x \phi_{m}^{2} \overline{e^{i(n q-m) \theta}}\left\langle e^{i(m-n q) \theta}\right\rangle_{b}
\end{aligned}
$$


For small $\kappa$, we can approximate both $\overline{e^{i(n q-m) \theta}}$ and $\left\langle e^{i(m-n q) \theta}\right\rangle_{b}$ by $J_{0}\left(2 \kappa \frac{x}{\Delta}\right)$. Carrying out the $\kappa$ and $x$ integration in Eq.(21) by noting that $\int_{0}^{\infty} d t J_{0}^{2}(t) e^{-\beta t^{2}} \simeq \frac{1}{\pi} \ln \beta^{-\frac{1}{2}}$, we have

$$
\int_{-\infty}^{\infty} d x \dot{\phi}_{n}^{m} \hat{\Upsilon}_{e}^{l}\left(e^{-i n q \theta} \phi_{n}\right\rangle_{b} \approx\left(\frac{\varepsilon}{2}\right)^{\frac{1}{2}}\left(1-\frac{\omega_{e}^{*}}{\omega}\right) g_{n} \hat{\phi}_{n}^{2} \Delta \ln \frac{x_{t}}{\Delta}
$$

Noting that $\varepsilon(\vec{k}, \omega) \approx i \gamma_{e}^{l} \frac{\partial}{\partial \omega} \varepsilon\left(\vec{k}, \omega_{\vec{k}}\right)$, and $\frac{\partial}{\partial \omega} \varepsilon=-\frac{\omega_{i}^{*}}{\omega^{2}}$, the linear growth rate is obtained by substituting Eq.(19) and Eq.(22) into Eq.(18),

$$
\frac{\gamma_{e}^{l}}{\omega_{\vec{k}}}=\left(\frac{\epsilon}{2}\right)^{\frac{1}{2}}\left(1-\frac{\omega_{\vec{k}}}{\omega_{e}^{*}}\right) \Delta \ln \left(\frac{x_{t}}{\Delta}\right) \operatorname{Im}\left(g_{n} \sqrt{\frac{i \mu}{\pi}}\right)
$$

Eq.(23) showes that both $\operatorname{Reg}_{n}$ and $I m g_{n}$ contribute to the linear growth rate, because $\sqrt{\frac{i \mu}{\pi}}=\sqrt{\frac{|\mu|}{2 \pi}}\left[1+i \operatorname{sign}\left(k_{\theta}\right)\right]$ is complex (where $\operatorname{sign}\left(k_{\theta}\right)$ is the sign function, equal to 1 for $k_{\theta}>0$ and -1 for $k_{\theta}<0$ ). This phenomenon is due to the fact that the eigenmode has outgoing wave structure and the trapped electron response and mode vary on different spatial scales. However, in the discussion of various trapped electron modes, the $\operatorname{Re}_{n}$ contribution to the linear growth rate can generally be neglected for the following two reasons. First, $\operatorname{Re}_{n}$ is smailer than $I m g_{n}$ in the dissipative regime. Second, the linear growth rate should be an even function of the poloidal wavenumber $\left(k_{\theta}\right)$. The contribution of $\operatorname{Reg}_{n}$ to the linear growth rate, which is an odd function of $k_{\theta}$, should be neglected even when $\operatorname{Re} g_{n}$ is not necessarily smaller than $\operatorname{Im} g_{n}$. By neglectıng term associated with $\operatorname{Re} g_{n}$ in Eq.(23), we finally have,

$$
\frac{\gamma_{e}^{\prime}}{\left|\omega_{\bar{k}}\right|}=\frac{1}{2}\left(\frac{\epsilon}{\pi}\right)^{\frac{1}{2}}\left(1-\frac{\omega_{\bar{k}}}{\omega_{e}^{*}}\right) \frac{\Delta}{x_{t}} \ln \left(\frac{x_{t}}{\Delta}\right) \operatorname{Im}\left(g_{n}\right) .
$$

The above equation clearly indicates that due to the radial localization of trapped electron response, the linear growth rate of the mode is reduced by a factor of $\frac{\Delta}{x_{t}} \ln \left(\frac{x_{1}}{\Delta}\right) \propto \sqrt{\frac{L_{n}}{L_{0}}}<1$. The instability source term $\operatorname{Im}\left(g_{n}\right)$ in the above equation can be calculated from Eq.(11) in various collisionality regime. The resilts are:

1.) Collisionless Regime $\left(\omega>\omega_{D_{e}}>\nu_{e f f}\right)$

$$
\operatorname{Im}\left(g_{n}\right)=2 \sqrt{\pi}\left(\frac{\omega}{\omega_{D e}}\right)^{\frac{3}{2}} e^{-\frac{\omega}{D_{e}}}
$$


2.) Dissipative Regime $\left(\nu_{e f f}>\omega>\omega_{D e}\right)$

$$
\operatorname{Im}\left(g_{n}\right)=\frac{4}{\sqrt{\pi}} \frac{|\omega|}{\nu_{e f f}}
$$

For typical tokamak parameters, it can be shown that the most unstable mode occurs at $k_{\theta} \rho, \sim 1$, and the growth rate is smaller than the real Erequency, i.e. $\frac{r_{c}^{\prime}}{\omega_{k}}<1$.

The important results of the linear analysis are summarized as follows. First, the linear eigenmode (slab-like) is characterized by three different spatial scale lengths $\Delta, x_{t}$, and $x_{i}$ $\left(\Delta<x_{t}<x_{i}\right)$. Since the eigenmode spectrum extends to $x_{i}, \Delta\left(k_{\|} v_{i}\right) \sim \omega$ which implies that the wave-particle auto-correlation time is comparable to the wave period. This is in contrast to the shearless case where the wave-particle auto-correlation time is much longer than the wave period (i.e. $\left.k_{\|} v_{i} \ll \omega\right)$. Thus, nonlinear wave-particle interaction is much stronger in sheared slab than in a shearless system, where nonlinear wave-wave interaction is the dominant process. Second, the trapped electron mode is a short wavelength, strongly dispersive drift wave. Third, trapped electron response is radially localized to a narrow region $x \leq \Delta\left(<x_{t}\right)$ near the mode ratioal surface.

\section{Nonlinear Ion and Trapped Electron Response}

In this section, weak turbulence theory is employed to calculate the nonlinear ion and trapped electron response. Since in the dissipative regime the trapped electron"mediated norlinear interaction is negligble, we focus on the collisionless regime in the following analysis. The dissipative effect can be easily incorporated into the final result. In the weak turbulence theory, the nonlinear evolution of the mode occurs on a mich slower time scale $\Delta \omega^{-1}$ than the linear mode oscillation $\omega^{-1}$, where the turbulent decorrelation rate $\Delta \omega \sim \gamma_{e}^{l} \ll \omega$. The ions and electrons are primarily fiuid-like, namely, they are not resonant with the drift wave. The turbulence spectrum can be decomposed into a sum of modes with frequency and mode structure described by linear theory. Nonlinearly, these modes can couple with each other in various ways. 
By treating $N^{i}$ and $N_{n}^{e}$ as perturbations, we can solve Eqs.(3) and (6) perturbatively, namely, we expand $h_{1}^{i}, \hat{h}_{n}^{e}$, and $\phi_{!}$as $h_{1}^{i}=h_{!}^{i(1)}+h_{!}^{i(2)}+h_{1}^{i(3)}+\cdots, \hat{h}_{n}^{e}=\hat{h}_{n}^{e(1)}+\hat{h}_{n}^{e(2)}+$ $\hat{h}_{n}^{e(3)}+\cdots$, and $\phi_{l}=\phi_{!}^{(1)}+\phi_{!}^{(2)}+\phi_{!}^{(3)} \cdots$ To the lowest order, we have the linear responses,

$$
\begin{aligned}
& h_{i}^{i(1)}=\frac{e}{T_{i}} F_{i} \frac{\omega-\omega_{i}^{*}}{\omega-k_{\|} v_{\|}} J_{0}\left(\frac{k_{\perp} v_{\perp}}{\Omega_{i}}\right) \phi_{!}^{(1)} \\
& \hat{h}_{n}^{e(1)}=-\frac{e}{T_{e}} F_{e} \frac{\omega-\omega_{e}^{*}}{\omega-\omega_{d e}}\left\langle e^{-i n q \theta} \phi_{n}^{(1)}\right\rangle_{b}
\end{aligned}
$$

where $\phi_{l}^{(1)}=\phi_{l}^{(1)} \delta\left(\omega-\omega_{\vec{k}}\right)$ is the linear eigenmode with $\omega_{\vec{k}}$ being the linear eigenfrequency.

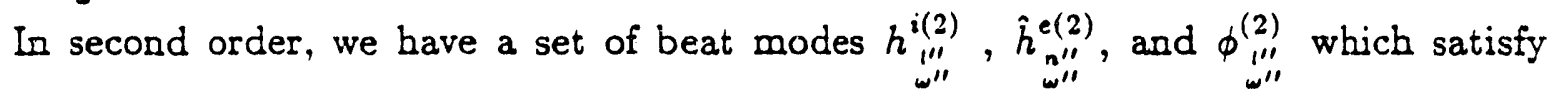
Eqs.(3) and (6), but are driven only by the direct interaction between the test mode $(l, \omega)$ and background mode $\left(l^{\prime}, \omega^{\prime}\right)$,

$$
\begin{aligned}
& h_{\substack{\prime \prime \prime \\
\omega^{\prime \prime}}}^{i(2)}=\frac{e}{T_{i}} F_{i} \frac{\omega^{\prime \prime}-\omega_{i}^{* \prime \prime}}{\omega^{\prime \prime}-k_{\|}^{\prime \prime} v_{\|}} J_{0}\left(\frac{k_{\perp}^{\prime \prime} v_{\perp}}{\Omega_{i}}\right) \phi_{\substack{\prime \prime \prime \\
\omega^{\prime \prime}}}^{(2)}+\frac{i}{\omega^{\prime \prime}-k_{\|}^{\prime \prime} v_{\|}} S_{\omega^{\prime \prime}}^{i \prime} \\
& \hat{h}_{\substack{n^{\prime \prime} \\
\omega^{\prime \prime}}}^{e(2)}=-\frac{e}{T_{e}} F_{e} \frac{\omega^{\prime \prime}-\omega_{e}^{\prime \prime}}{\omega-\omega_{d e}^{\prime \prime}}\left\langle e^{-i n^{\prime \prime} q \theta} \phi_{\substack{n^{\prime \prime} \\
\omega^{\prime \prime}}}^{(2)}\right\rangle_{b}+\frac{i}{\omega^{\prime \prime}-\omega_{d e}^{\prime \prime}} S_{\substack{n^{\prime \prime} \\
\omega^{\prime \prime}}}^{e}
\end{aligned}
$$

where the driving terms $S_{\substack{\prime \prime \prime \\ u_{\prime \prime}}}^{i}$ and $S_{\substack{n^{\prime \prime} \\ u^{\prime \prime}}}^{e}$ are given by,

$$
\begin{aligned}
& S_{{ }^{\prime \prime \prime}}^{i \prime \prime}=i \frac{c}{B}\left\{k_{\theta} J_{0}\left(\frac{k_{\perp} v_{\perp}}{\Omega_{i}}\right) \phi_{!}^{(1)} \frac{\partial}{\partial r} h_{L^{\prime}}^{i(1)}+k_{\theta}^{\prime} J_{0}\left(\frac{k_{\perp}^{\prime} v_{\perp}}{\Omega_{i}}\right) \phi_{\iota^{\prime}}^{(1)} \frac{\partial}{\partial r} h_{!}^{i(1)}\right. \\
& \left.-k_{\theta} h_{i}^{i(1)} \frac{\partial}{\partial r}\left[J_{0}\left(\frac{k_{\perp}^{\prime} v_{\perp}}{\Omega_{i}}\right) \phi_{\substack{\prime^{\prime} \\
w^{\prime}}}^{(1)}\right]-k_{\theta}^{\prime} h_{\substack{\prime^{\prime} \\
w^{\prime}}}^{(1)} \frac{\partial}{\partial r}\left[J_{0}\left(\frac{k_{\perp} v_{\perp}}{\Omega_{i}}\right) \dot{\phi}_{!}^{(1)}\right]\right\} \\
& S_{\substack{n^{\prime \prime} \\
n^{\prime \prime}}}^{e}=i \frac{c}{B}\left\{\frac{n q}{r}\left\langle e^{-i n q \theta} \phi_{n}^{(1)}\right\rangle_{b} \frac{\partial}{\partial r} \hat{h}_{\substack{n^{\prime} \\
w^{\prime}}}^{e(1)}-\frac{n q}{r}\left(\frac{\partial}{\partial r}\left\langle e^{-i n^{\prime} q \theta} \phi_{\substack{n^{\prime} \\
u^{\prime}}}^{(1)}\right\rangle_{b}\right) \hat{h}_{n}^{e(1)}\right. \\
& \left.+\frac{n^{\prime} q}{r}\left\langle e^{-i n^{\prime} q \theta} \phi_{\substack{n^{\prime} \\
s^{\prime}}}^{(1)}\right\rangle_{b}\right) \frac{\partial}{\partial r} \hat{h}_{n}^{e(1)}-\frac{n^{\prime} q}{r}\left(\frac{\partial}{\partial r}\left\langle e^{-i n q \theta} \phi_{n}^{(1)}\right\rangle_{b}\right) \hat{h}_{\substack{n^{\prime} \\
u^{\prime}}}^{e(1)}
\end{aligned}
$$

The second order ion and electron density perturbation can be obtained from Eqs.(4) and (7) by integrating $h_{\substack{m^{\prime \prime} \\ n^{\prime \prime}}}^{i(2)}$ and $\hat{h}_{\substack{m{ }^{\prime \prime} \\ n^{\prime \prime}}}^{e(2)}$ over velocity. To perform the velocity integration for ions, we neglect the resonance effect of the normal modes $(l, \omega)$ and $\left(l^{\prime}, \omega^{\prime}\right)$ in favor of the beat mode $\left(l^{\prime \prime}, \omega^{\prime \prime}\right)$ resonance, i.e. the ions are fluid-like relative to the normal modes $\omega \gg k_{\|} v_{i}$, and $\omega^{\prime} \gg k_{\|}^{\prime} v_{i}$. but are dissipative relative to the teat modes $\omega^{\prime \prime} \leq k_{\|}^{\prime \prime} v_{i}$. The results are

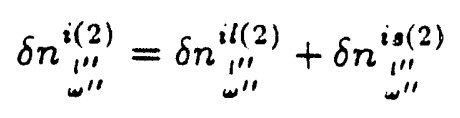




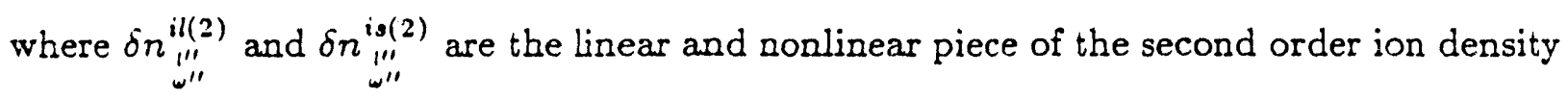
response, and given by

$$
\begin{aligned}
& \delta n_{\substack{i \prime \prime \\
\omega^{\prime \prime}}}^{i(2)}=-\frac{e}{T_{i}} N\left[1+\left(1-\frac{\omega_{i}^{*^{\prime \prime}}}{\omega^{\prime \prime}}\right) \frac{\omega^{\prime \prime}}{\left|k_{\|}^{\prime \prime} v_{i}\right|} Z\left(\frac{\omega^{\prime \prime}}{\left|k_{\|}^{\prime \prime} v_{i}\right|}\right) \Gamma_{0}\left(b_{i}^{\prime \prime}\right)\right] \phi_{\substack{\prime \prime \prime \\
\omega^{\prime \prime}}}^{(2)} \\
& \delta n_{\substack{\prime s(2) \\
{ }^{\prime \prime}}}^{i \prime \prime}=\frac{e}{T_{e}^{\prime}} N \frac{c}{B}\left(\frac{\omega_{e}^{*^{\prime}}}{\omega^{\prime}}-\frac{\omega_{e}^{*}}{\omega}\right) \frac{\left\langle J_{0}\left(k_{\perp} \rho_{i}\right) J_{0}\left(k_{\perp}^{\prime} \rho_{i}\right) J_{0}\left(k_{\perp}^{\prime \prime} \rho_{i}\right)\right\rangle_{\perp}}{\left|k_{\|}^{\prime \prime} v_{i}\right|} Z\left(\frac{\omega^{\prime \prime}}{\left|k_{\|}^{\prime \prime} v_{i}\right|}\right) \\
& \left(k_{\theta} \dot{\phi}_{!}^{(1)} \frac{\partial}{\partial r} \dot{\phi}_{\substack{\prime \prime \\
w^{\prime}}}^{(1)}-k_{\theta}^{\prime} \dot{\phi}_{\omega^{\prime}}^{(1)} \frac{\partial}{\partial r} \phi_{!}^{(1)}\right)
\end{aligned}
$$

where $b_{i}^{\prime \prime}=\frac{1}{2} k_{\perp}^{\prime \prime 2} \rho_{i}^{2}$, and

$$
\left\langle J_{0}\left(k_{\perp} \rho_{i}\right) J_{0}\left(k_{\perp}^{\prime} \rho_{i}\right) J_{0}\left(k_{\perp}^{\prime \prime} \rho_{i}\right)\right\rangle_{\perp} \equiv 2 \int_{0}^{\infty} x_{\perp} d x_{\perp} e^{-x_{\perp}^{2}} J_{0}\left(k_{\perp} \rho_{i} x_{\perp}\right) J_{0}\left(k_{\perp}^{\prime} \rho_{i} x_{\perp}\right) J_{0}\left(k_{\perp}^{\prime \prime} \rho_{i} x_{\perp}\right)
$$

The velocity integrated trapped electron population can be obtained in a similar way. For most trapped electrons, we have $\omega \gg \omega_{d e}$ and $\omega^{\prime} \gg \omega_{d e}^{\prime}$, hence only a small number of high energy particles in the distribution tail can be resonant with the primary wave. However, since the beating mode frequency $\omega^{\prime \prime}$ can become smaller than either $\omega$ or $\omega^{\prime}$, the bulk of the trapped electron distribution can be resonant with the beat wave. Thus, by retaining only the beat mode resonance effect in the trapped $\epsilon$ lectron velocity integral, we have

$$
\delta n_{\substack{\prime \prime \prime \\ \prime \prime \prime}}^{e(2)}=\delta r^{\prime \prime}{ }_{\substack{\prime \prime \prime \\ w^{\prime \prime}}}^{e l(2)}+\delta n_{\substack{\prime \prime \prime \\ \prime \prime \prime}}^{e s(2)}
$$

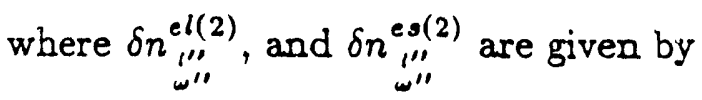

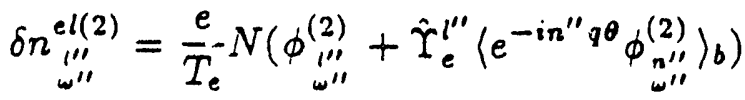

$$
\begin{aligned}
& \hat{\Upsilon}_{e}^{l^{\prime \prime}}\left\langle e^{-i n^{\prime \prime} g \theta} \phi_{\substack{n^{\prime \prime} \\
\omega^{\prime \prime}}}^{(2)}\right\rangle_{b}=2\left(\frac{\epsilon}{2}\right)^{\frac{1}{2}} g_{n^{\prime \prime}} \int_{0}^{1} d \kappa^{2} \overline{e^{i\left(n^{\prime \prime} q-m^{\prime \prime}\right) \theta}}\left\langle e^{-i n^{\prime \prime} q \theta} \phi_{\substack{n^{\prime \prime} \\
\omega^{\prime \prime}}}^{(2)}\right\rangle_{b} \\
& g_{n^{\prime \prime}}=\left(\frac{\omega_{e}^{*^{\prime \prime}}}{\omega^{\prime \prime}}-1\right) \frac{\omega^{\prime \prime}}{\omega_{D e}^{\prime \prime}}\left[1+\sqrt{\frac{\omega^{\prime \prime}}{\omega_{D e}^{\prime \prime}}} Z\left(\sqrt{\frac{\omega^{\prime \prime}}{\omega_{D e}^{\prime \prime}}}\right)\right]
\end{aligned}
$$

and

$$
\delta n_{\substack{\prime^{\prime \prime} \\ u^{\prime \prime}}}^{e s(2)}=2 \frac{e}{T_{e}} N\left(\frac{\epsilon}{2}\right)^{\frac{1}{2}} \frac{c}{B}\left(\frac{\omega_{e}^{*^{\prime}}}{\omega^{\prime}}-\frac{\omega_{e}^{*}}{\omega}\right) G_{n, n^{\prime}}^{e} \int_{0}^{1} d \kappa^{2} \overline{e^{i\left(n^{\prime \prime} q-m^{\prime \prime}\right) \theta}}
$$




$$
\begin{gathered}
\times\left[\frac{n q}{r}\left\langle e^{-i n q \theta} \phi_{n}^{(1)}\right\rangle_{b} \frac{\partial}{\partial r}\left\langle e^{-i n^{\prime} q \theta} \phi_{\substack{n^{\prime} \\
\omega^{\prime}}}^{(1)}\right\rangle_{b}-\frac{n^{\prime} q}{r}\left\langle e^{-i n^{\prime} q \theta} \phi_{\substack{n^{\prime} \\
\omega^{\prime}}}^{(1)}\right\rangle_{b} \frac{\partial}{\partial r}\left\langle e^{-i n q \theta} \phi_{\substack{n \\
\omega}}^{(1)}\right\rangle_{b}\right] \\
G_{n, n^{\prime}}^{e}=\frac{1}{\omega_{D e}^{\prime \prime}}\left[1+\sqrt{\frac{\omega^{\prime \prime}}{\omega_{D e}^{\prime \prime}}} Z\left(\sqrt{\frac{\omega^{\prime \prime}}{\omega_{D e}^{\prime \prime}}}\right)\right]
\end{gathered}
$$

By imposing the quasineutrality condition, we can obtain an equation that determines the second order potential perturbation $\phi_{\substack{\prime \prime \prime \\ \prime \prime \prime}}^{(2)}$,

$$
\begin{aligned}
& {\left[1+\tau+\tau\left(1-\frac{\omega_{i}^{*^{\prime \prime}}}{\omega^{\prime \prime}}\right) \frac{\omega^{\prime \prime}}{\left|k_{\|}^{\prime \prime} v_{i}\right|} \Gamma_{0}\left(b_{i}^{\prime \prime}\right) Z\left(\frac{\omega^{\prime \prime}}{\left|k_{\|}^{\prime \prime} v_{i}\right|}\right)\right] \phi_{\substack{\prime \prime \prime \\
\omega^{\prime \prime}}}^{(2)}+\hat{\Upsilon}^{\prime}\left\langle e^{-i n^{\prime \prime} q \theta} \phi_{n^{\prime \prime}}\right\rangle_{b}} \\
& =\hat{N}_{\substack{\prime \prime \prime \\
\omega^{\prime \prime}}}^{i(s)}-\hat{N}_{\frac{{ }^{\prime \prime}}{e(s)}}^{(s)}
\end{aligned}
$$

where

$$
\begin{aligned}
& \hat{N}^{i(s)}=\frac{T_{e}}{e N} \delta n^{\prime \prime \prime}{ }_{\substack{\prime \prime \prime \\
s^{\prime \prime}}}^{i s(2)} \\
& \hat{N}_{\substack{l \prime \prime \\
\omega^{\prime \prime}}}^{e(s)}=\frac{T_{e}}{e N} \delta n_{\substack{l \prime \prime \\
\omega^{\prime \prime}}}^{e s(2)}
\end{aligned}
$$

The left side of Eq.(32) is simply the linear dielectric for the beat mode $\left(\vec{k}^{\prime \prime}, \omega^{\prime \prime}\right)$. The terms on the right side represent the drive by the direct interaction between the test mode $(\vec{k}, \omega)$ and the background mode $\left(\vec{k}^{\prime}, \omega^{\prime}\right)$ from both ion and trapped electron nonlinearity. Clearly, Eq.(32) is a very complicated, inhomogenious integral-cifferential equation which is not amenable to analytical solution. Nevertheless, some important properties of $\phi_{t^{\prime \prime \prime}}^{(2)}$

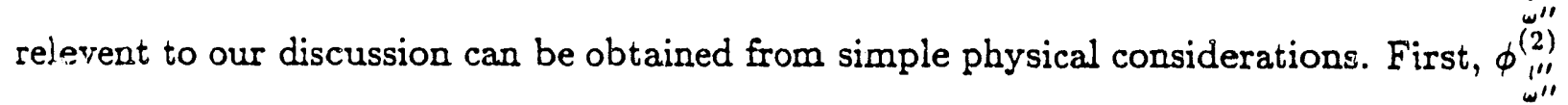
represents a process of nonlinear three wave resonance interaction (decay type interaction), i.e., $\phi_{w^{\prime \prime}}^{(2)} \propto \frac{1}{\epsilon\left(\bar{k}^{\prime \prime}, \omega^{\prime \prime}\right)}$. The three wave resonance interaction is severely restricted by the dispersion of the primary wave. Second, in the presence of magnetic shear, the beat mode is intrinsically dissipative in nature (i.e., heavily Landau damped for $\omega+\omega^{\prime} \leq k_{\|}^{\prime \prime} v_{i}$ ). In oider to see this, we note that the spectrum of the Pearlstein-Berk eigenmode extends to

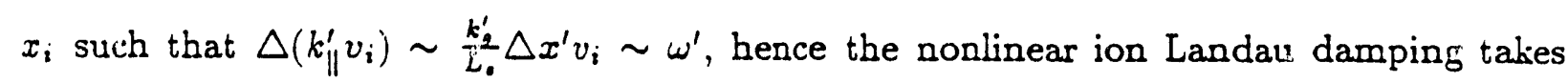
place when $\omega+\omega^{\prime} \leq \Delta\left(k_{\|}^{\prime \prime} v_{i}\right) \sim \omega^{\prime}$. Therefore, in a sheared magnetic field, the range of frequency of the background mode which can interact with the test mode through ion Compton scattering is very broad. This is in contrast to the shearless case where the 
condition that the ion is fluid-like $\omega \gg k_{\|} v_{i}$ implies that $\omega+\omega^{\prime}<k_{\|}^{\prime} v_{i} \ll \omega^{\prime}$ for beat mode interaction, i.e. without shear, the beat interaction is severely restricted. The strong dissipative character of the beat mode in a sheared magnetic field renders $\phi^{(2)}$ (2) $^{\prime \prime \prime}$ significant only in a region of width $x_{i}^{\prime \prime}$ which is much narrow than the spatial width of the linear eigenmode spectrum $x_{i}^{\prime}\left(\right.$ i.e. $\left.x_{i}^{\prime \prime} \ll x_{i}^{\prime}\right)$. As a consequence, the oft-referred-to cancellation of the shielding and bare scattering contributions to the order of $\circ\left(k_{\perp}^{2} \rho_{i}^{2}\right)$, which occurs in the weak turbulence theory of shearless drift wave turbulence, does not occur in a sheared system.

Now, following standard proceedures ${ }^{13}$, and making use of Eqs.(27a) and (27b), we have the third order perturbation in both $h_{1}^{i}$ and $\underset{n}{e}$,

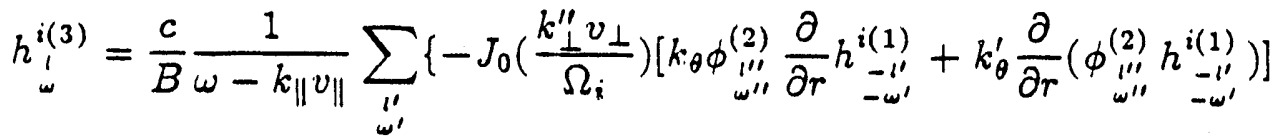

$$
\begin{aligned}
& +\frac{e}{T_{e}} F_{i} J_{0}\left(\frac{k_{\perp}^{\prime \prime} v_{\perp}}{\Omega_{i}}\right) J_{0}\left(\frac{k_{\perp}^{\prime} v_{\perp}}{\Omega_{i}}\right)\left[k_{\theta} \frac{\omega^{\prime \prime}-\omega_{i}^{*^{\prime \prime}}}{\omega^{\prime \prime}-k_{\|}^{\prime \prime} v_{\|}} \phi_{\prime^{\prime \prime}}^{(2)} \frac{\partial}{\partial r} \phi_{\substack{-\omega^{\prime \prime} \\
-\omega^{\prime}}}^{(1)}+k_{\theta}^{\prime} \frac{\partial}{\partial r}\left(\frac{\omega^{\prime \prime}-\omega_{i}^{*^{\prime \prime}}}{\omega^{\prime \prime}-k_{\|}^{\prime \prime} v_{\|}} \phi_{\substack{\prime \prime \prime \\
\omega^{\prime \prime}}}^{(2)} \phi_{\substack{\prime^{\prime \prime} \\
-\omega^{\prime}}}^{(1)}\right)\right] \\
& \left.+J_{0}\left(\frac{k_{\perp}^{\prime} v_{\perp}}{\Omega_{i}}\right)\left[\frac{i k_{\theta} S_{{ }^{\prime \prime}}^{i \prime}}{\omega^{\prime \prime}-k_{\|}^{\prime \prime} v_{\|}} \frac{\partial}{\partial r} \phi_{\substack{-\prime^{\prime \prime} \\
-w^{\prime}}}^{(1)}+\frac{\partial}{\partial r}\left(\frac{i k_{\theta}^{\prime} S_{\prime^{\prime \prime}}^{i}}{\omega^{\prime \prime}-k_{\|}^{\prime \prime} v_{\|}} \phi_{\substack{-w^{\prime \prime} \\
-\omega^{\prime}}}^{(1)}\right)\right]\right\}
\end{aligned}
$$

and

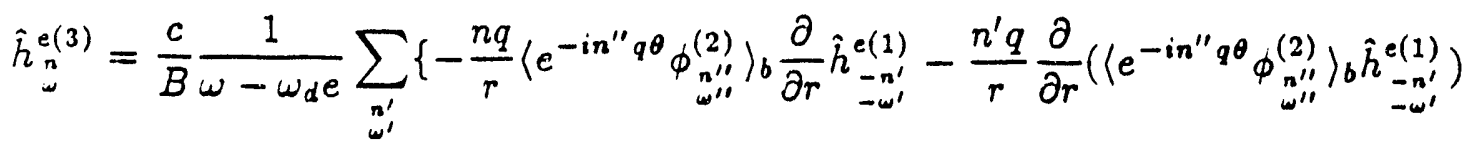

$$
\begin{aligned}
& -\frac{e}{T_{e}} F_{e} \frac{\omega^{\prime \prime}-\omega_{e}^{*^{\prime \prime}}}{\omega^{\prime \prime}-\omega_{d e}^{\prime \prime}}\left[\frac{n q}{r}\left\langle e^{-i n^{\prime \prime} q \theta} \phi_{\substack{n^{\prime \prime} \\
w^{\prime \prime}}}^{(2)}\right\rangle_{b} \frac{\partial}{\partial r}\left\langle e^{i n^{\prime} q \theta} \phi_{\substack{-n^{\prime} \\
-\omega^{\prime}}}^{(1)}\right\rangle_{b}+\frac{n^{\prime} q}{r} \frac{\partial}{\partial r}\left(\left\langle e^{-i n^{\prime \prime} q \theta} \phi_{\substack{n^{\prime \prime} \\
u^{\prime \prime}}}^{(2)}\right\rangle_{b}\left\langle e^{i n^{\prime} q \theta} \phi_{\substack{n^{\prime} \\
-\omega^{\prime}}}^{(1)}\right\rangle_{b}\right)\right] \\
& \left.+\frac{i}{\omega^{\prime \prime}-\omega_{d e}^{\prime \prime}}\left[\frac{n q}{r} S_{\substack{n^{\prime \prime} \\
\prime^{\prime \prime}}}^{e} \frac{\partial}{\partial r}\left\langle e^{i n^{\prime} q \theta} \phi_{\substack{-n^{\prime} \\
-\omega^{\prime}}}^{(1)}\right\rangle_{b}+\frac{n^{\prime} q}{r} \frac{\partial}{\partial r}\left(S_{\substack{n^{\prime \prime} \\
\omega^{\prime \prime}}}^{e}\left\langle e^{i n^{\prime} q \theta} \phi_{\substack{-n^{\prime} \\
-\omega^{\prime}}}^{(1)}\right\rangle_{b}\right)\right]\right\}
\end{aligned}
$$

On the right sicle of the above equations, the first two terms that involve $\phi^{(2)}$ (2) $^{\prime \prime \prime}$ or $\phi_{\substack{\prime \prime \prime \\ n^{\prime \prime}}}^{(2)}$ represent nonlincar three wave resonant interaction effects. The last two terms that involve the resonance factor $\frac{1}{\omega^{\prime \prime}-k_{\|}^{\prime \prime} v_{\|}}$or $\frac{1}{\omega^{\prime \prime}-\omega_{d e}^{\prime \prime}}$ represent the effect of scattering of waves by bare particles, namely, the ion Compton scattering and trapped electron Cumpton scattering (nonlinear trapped electron-wave interaction). The second two terms that involve both 
$\phi_{\mathbf{\omega}^{\prime \prime}}^{(2)}$, and the resonance factor represent the effect of scattering of waves by the shielding or polarization cloud generated by a moving charged particle. For slab-like trapped electron drift modes, the three wave resonance interaction is irrelevent since the most unstable modes are short wavelength dispersive waves $\left(k_{\theta} \rho_{3} \approx 1\right)$ so that the beat mode is not resonant. The effect of the scattering of waves by the shielding cloud is weat because the second order potential perturbation $\phi_{\text {(') }}^{(2)}$ is radially localized to a region which is much more narrower than the linear mode width $\left(x_{i}^{\prime \prime} \ll x_{i}^{\prime}\right)$. Therefore, in a sheared magnetic field, the dominarit nonlinear process is the bare particle induced wave scattering. In the following discussion, terms associated with $\phi_{\substack{\prime \prime \prime \\ \prime \prime \prime}}^{(2)}$ will be neglected. By making use of Eqs.(28a) and (28b), and keeping only the beat-mode-particle resonance effect, we have the third order ion and trapped electron response,

$$
\begin{aligned}
& h_{m}^{i(3)}=-k_{\theta}^{2} \mu_{\theta \theta}^{i} J_{0}\left(\frac{k_{\perp} v_{\perp}}{\Omega_{i}}\right) \phi_{n}+\mu_{r r}^{i} J_{0}\left(\frac{k_{\perp} v_{\perp}}{\Omega_{i}}\right) \frac{\partial^{2}}{\partial r^{2}} \phi_{n} \\
& \hat{h}_{n}^{e(3)}=-\left(\frac{n q}{r}\right)^{2} \mu_{\theta \theta}^{e}\left\langle e^{-i n q \theta} \phi_{n}\right\rangle_{b}+\mu_{r r}^{e} \frac{\partial^{2}}{\partial r^{2}}\left\langle e^{-i n q \theta} \phi_{n}\right\rangle_{b}
\end{aligned}
$$

where the spectrum dependent quantities are

$$
\begin{aligned}
& \mu_{\theta \theta}^{i}=\left(\frac{c}{B}\right)^{2} \frac{1}{\omega} \sum_{\substack{m^{\prime} \\
n^{\prime}}} R_{\substack{m^{\prime \prime} \\
n^{\prime \prime}}} J_{0}^{2}\left(\frac{k_{\perp}^{\prime} v_{\perp}}{\Omega_{i}}\right)\left|\frac{\partial}{\partial r} \phi_{\substack{n^{\prime} \\
n^{\prime}}}\right|^{2} \frac{e}{T_{i}} F_{i}\left(\frac{\omega_{i}^{*}}{\omega}-\frac{\omega_{i}^{*^{\prime}}}{\omega^{\prime}}\right) \\
& \mu_{r r}^{i}=\left(\frac{c}{B}\right)^{2} \frac{1}{\omega} \sum_{\substack{m^{\prime} \\
n^{\prime}}} R_{m_{n^{\prime \prime}}} k_{\theta}^{\prime 2} J_{0}^{2}\left(\frac{k_{\perp}^{\prime} v_{\perp}}{\Omega_{i}}\right)\left|\phi_{\substack{m^{\prime} \\
n^{\prime}}}\right|^{2} \frac{e}{T_{i}} F_{i}\left(\frac{\omega_{i}^{*}}{\omega}-\frac{\omega_{i}^{*^{\prime}}}{\omega^{\prime}}\right) \\
& R_{\theta \theta}^{e}=\left(\frac{c}{B}\right)^{2} \frac{1}{\omega} \sum_{n^{\prime}} R_{n^{\prime \prime}}\left|\frac{\partial}{\partial r}\left\langle e^{-i n^{\prime} q \theta} \phi_{n^{\prime}}\right\rangle_{b}\right|^{2} \frac{e}{T_{e}} F_{e}\left(\frac{\omega_{e}^{m^{\prime}}}{\omega^{\prime}}-\frac{\omega_{e}^{*}}{\omega}\right) \\
& \mu_{r r}^{e}=\left(\frac{c}{B}\right)^{2} \frac{1}{\omega} \sum_{n^{\prime}} R_{n^{\prime \prime}}\left(\frac{n^{\prime} q}{r}\right)^{2}\left|\left\langle e^{-i n^{\prime} q \theta} \phi_{n^{\prime}}\right\rangle_{b}\right|^{2} \frac{e}{T_{e}} F_{e}\left(\frac{\omega_{e}^{*^{\prime}}}{\omega^{\prime}}-\frac{\omega_{e}^{*}}{\omega}\right) \\
& R_{n^{\prime \prime}}=\frac{1}{\omega^{\prime \prime}-\omega_{d e}^{\prime \prime}}
\end{aligned}
$$

In arriving at the above results, the fact that the linear modes are densely packed has been ultilized so that only terms with even parity in $x^{\prime}$ are retained, while terms with odd 
parity in $x^{\prime}$ like $\psi_{\substack{\prime^{\prime} \\-w^{\prime}}}^{(1)} \frac{\partial}{\partial r} \psi_{w^{\prime}}^{(1)}$ and $\left\langle e^{i n^{\prime} q \theta} \phi_{\substack{w^{\prime} \\-w^{\prime}}}^{(1)}\right\rangle_{b} \frac{\partial}{\partial r}\left\langle e^{-i n^{\prime} q \theta} \phi_{\substack{n^{\prime} \\ w^{\prime}}}^{(1)}\right\rangle_{b}$ vanish. The superfluous subscripts $\omega, \omega^{\prime}$ on all the fluctuating quantities have been dropped, since they are related to the wavenumbers $n$ and $n^{\prime}$ through the linear dispersion relation $\omega=\omega_{\vec{k}}$, and $\omega^{\prime}=\omega_{\bar{k}^{\prime}}$. Without any confusion, $\phi_{n}^{(1)}$, and $\phi_{\substack{\prime^{\prime} \\ u^{\prime}}}^{(1)}$ have been replaced by $\phi_{n}$ and $\phi_{\substack{m^{\prime} \\ n^{\prime}}}$ to represent the linear eigenmode. The ion density response up to third order is then given by

$$
\begin{aligned}
& \delta n_{n}^{i} \underset{n}{i}=-\frac{e}{T_{e}} N \phi_{n}^{m}+\int d^{3} \vec{v} J_{0}\left(\frac{k_{\perp} v_{\perp}}{\Omega_{i}}\right)\left(h_{n}^{i(1)}+h_{\substack{n \\
n}}^{i(3)}\right) \\
& =\delta n_{n}^{i(1)}+\delta n_{n}^{i(3)}
\end{aligned}
$$

where $\delta n_{n}^{i(1)}$ is the linear ion density response given by Eq.(12), $\delta n_{n}^{i(3)}$ is the third order ion density response and can be obtained by integrating Eq.(43) over velocity space,

$$
\delta n_{n}^{i(3)}=\frac{e}{T_{e}} N\left[-k_{\theta}^{2} D_{\theta \theta}^{i}+D_{r r}^{i} \frac{\partial^{2}}{\partial r^{2}}\right] \phi_{n}^{m}
$$

where

$$
\begin{aligned}
& D_{\theta \theta}^{i}=\frac{1}{\omega}\left(\frac{c}{B}\right)^{2} \sum_{\substack{m^{\prime} \\
n^{\prime}}}\left|\frac{\partial}{\partial r} \phi_{\substack{m^{\prime} \\
n^{\prime}}}\right|^{2}\left(\frac{\omega_{e}^{*}}{\omega}-\frac{\omega_{e}^{*^{\prime}}}{\omega^{\prime}}\right) G_{m n, m^{\prime} n^{\prime}}^{i} \\
& D_{r r}^{i}=\frac{1}{\omega}\left(\frac{c}{B}\right)^{2} \sum_{\substack{m^{\prime} \\
n^{\prime}}} k_{\theta}^{\prime 2}\left|\phi_{\substack{m^{\prime} \\
n^{\prime}}}\right|^{2}\left(\frac{\omega_{e}^{*}}{\omega}-\frac{\omega_{e}^{*^{\prime}}}{\omega^{\prime}}\right) G_{m n, m^{\prime} n^{\prime}}^{i}
\end{aligned}
$$

The interaction kernel $G_{m n, m^{\prime} n^{\prime}}^{i}$ is

$$
G_{m n, m^{\prime} n^{\prime}}^{i}=\left\langle J_{0}^{2}\left(k_{\perp} \rho_{i}\right) J_{0}^{2}\left(k_{\perp}^{\prime} \rho_{i}\right)\right\rangle_{\perp} \frac{1}{\left|k_{\|}^{\prime \prime} v_{i}\right|} Z\left(\frac{\omega^{\prime \prime}}{\left|k_{\|}^{\prime \prime} v_{i}\right|}\right)
$$

where

$$
\left\langle J_{0}^{2}\left(k_{\perp} \rho_{i}\right) J_{0}^{2}\left(k_{\perp}^{\prime} \rho_{i}\right)\right\rangle_{\perp}=2 \int_{0}^{\infty} x_{\perp} d x_{\perp} e^{-x_{\perp}^{2}} J_{0}^{2}\left(k_{\perp} \rho_{i} x_{\perp}\right) J_{0}^{2}\left(k_{\perp}^{\prime} \rho_{i} x_{\perp}\right) .
$$

The electron density response up to third order is given by

$$
\begin{aligned}
\delta n_{\substack{m \\
m}}^{e} & =\frac{e}{T_{e}} N \phi_{n}^{m}+\int_{-\pi}^{\pi} \frac{d \theta}{2 \pi} e^{i(n q-m) \theta} \int_{t} d^{3} \vec{v}\left(\hat{h}_{n}^{e(1)}+\hat{h}_{n}^{e(3)}\right) \\
& =\delta n_{\substack{m \\
n}}^{e(1)}+\delta n_{n}^{e(3)}
\end{aligned}
$$


where $\delta n_{n}^{e(1)}$ is the linear electron response, given by $E c_{1},(10 a)$ and $(10 b), \delta n_{n}^{e(3)}$ is the nonlinear trapped electron response and can be obtained by using Eq.(34b),

$$
\begin{aligned}
\delta n_{n}^{e(3)} & =\frac{e}{T_{e}} N \hat{\Upsilon}_{e}^{n l}\left\langle e^{-i n q \theta} \phi_{n}\right\rangle_{b} \\
\hat{\Upsilon}_{e}^{n !}\left\langle e^{-i n q \theta} \phi_{n}\right\rangle_{b} & =\left(\frac{\epsilon}{2}\right)^{\frac{1}{2}} \int_{0}^{1} d \kappa^{2} \overline{e^{i(n q-m)} \bar{\theta}}\left[-\left(\frac{n q}{r}\right)^{2} D_{\theta \theta}^{e}+D_{r r}^{e} \frac{\partial^{2}}{\partial r^{2}}\right]\left\langle e^{-i n g \theta} \phi_{n}\right\rangle_{b}
\end{aligned}
$$

with

$$
\begin{aligned}
& D_{\theta \theta}^{e}=\frac{2}{\omega}\left(\frac{c}{B}\right)^{2} \sum_{n^{\prime}}\left|\frac{\partial}{\partial r}\left\langle e^{-i n^{\prime} q \theta} \phi_{n^{\prime}}\right\rangle_{b}\right|^{2}\left(\frac{\omega_{e}^{*}}{\omega}-\frac{\omega_{e}^{*^{\prime}}}{\omega^{\prime}}\right) G_{n, n^{\prime}}^{e} \\
& D_{r r}^{e}=\frac{2}{\omega}\left(\frac{c}{B}\right)^{2} \sum_{n^{\prime}}\left(\frac{n^{\prime} q}{r}\right)^{2}\left|\left\langle e^{-i n^{\prime} q \theta} \phi_{n^{\prime}}\right\rangle_{b}\right|^{2}\left(\frac{\omega_{e}^{*}}{\omega}-\frac{\omega_{e}^{*^{\prime}}}{\omega^{\prime}}\right) G_{n, n^{\prime}}^{e}
\end{aligned}
$$

\section{Wave Kinetic Equation for Trapped Electron Mode Turbulence}

In this section, we derive a wave kinetic equation which describes the nonlinear evolution of the fluctuation spectrum using the previously derived nonlinear responses. By imposing the quasineutıality condition $\delta n_{n}^{e} \underset{n}{e}=\delta n_{n}^{i}$ for the nonlinear responses, we obtain a nonlinear eigenmode equation

$$
\begin{aligned}
& \left\{\rho_{s}^{2} \frac{\partial^{2}}{\partial r^{2}}+\frac{\omega_{e}^{*}}{\omega}-k_{\theta}^{2} \rho_{s}^{2}-1+\mu^{2} \rho_{s}^{2} x^{2}\right\} \phi_{n}^{m} \\
= & \left(\hat{\Upsilon}_{e}^{l}+\hat{\Upsilon}_{e}^{n l}\right)\left\langle e^{-i n q \theta} \phi_{n}\right\rangle_{b}+\left(k_{\theta}^{2} D_{\theta \theta}^{i}-D_{r r}^{i} \frac{\partial^{2}}{\partial r^{2}}\right) \phi_{n}^{m}
\end{aligned}
$$

To solve this equation, techniques in solving the linear eigenmode equation are again ultilized, i.e. we treat the terms on the right side of Eq.(42) as perturbations. This is possible because in the weak turbulence theory, the nonlinear interaction is at most comparable to the linear growth rate. In lowest order, we have the outgoing wave solution for the most unstable (least shear damped) mode,

$$
\phi_{n}^{(0)}=\hat{\phi}_{n}^{m} e^{-\frac{i n x^{2}}{2}}
$$

the corresponding real frequency,

$$
\omega_{\vec{k}}=\frac{\omega_{e}^{*}}{1+k_{\theta}^{2} \rho_{s}^{2}}
$$


and the shear damping rate

$$
\gamma^{s d}=-\frac{L_{n}}{L_{n}}\left|\omega_{\vec{k}}\right|
$$

To the next order, from perturbation theory we have,

$$
\varepsilon(\vec{k}, \omega) \int_{-\infty}^{\infty} d x\left(\phi_{n}^{(0)}\right)^{2}=I_{e}^{l}+I_{e}^{n l}+I_{i}^{n l}
$$

where $\omega=\omega_{\vec{k}}+i \gamma$, and $I_{e}^{l}, I_{e}^{n l}$ and $I_{i}^{n l}$ are defined by the following integrals,

$$
\begin{aligned}
& I_{e}^{l}=\int_{-\infty}^{\infty} d x \phi_{\substack{m \\
m}}^{(0)} \hat{\Upsilon}_{e}^{l}\left\langle e^{-i n q \theta} \phi_{n}^{(0)}\right\rangle_{b} \\
& I_{e}^{n l}=\int_{-\infty}^{\infty} d x \phi_{m}^{(0)} \hat{\Upsilon}_{e}^{n l}\left\langle e^{-i n q \theta} \phi_{n}^{(0)}\right\rangle_{b} \\
& I_{i}^{n l}=\int_{-\infty}^{\infty} d x\left\{k_{\theta}^{2} D_{\theta \theta}^{i(0)}(\underset{\substack{m \\
m}}{(0)})^{2}+D_{r i^{*}}^{i(0)}\left(\frac{\partial}{\partial r} \phi_{\substack{m \\
m}}^{(0)}\right)^{2}\right\}
\end{aligned}
$$

It is interesting to note that the above integrals involve $\underset{n}{\phi_{n}^{0}}{ }^{2}$ rather than $\left.\underset{n}{\mid \phi_{n}^{0}}\right|^{2}$. Therefore, it is expected that the principal contribution to the integrals is from the region $x<x_{t}$ where the mode is non-oscillatory. Outside this region, rapid cancelation in the integration occurs because of the fast oscillation character of $\phi_{n}^{0}{ }_{n}^{2}$ for $x>x_{t}$. Physically, this can be interpreted as that only the energy input or outtlow in region $x<x_{t}$ is relevent to the growth or damping of the mode. The first integral $I_{e}^{l}$ has been evaluated in Sec.III (Eq.(22)). By noting that for $x_{t}>\Delta$, we have $\frac{\partial}{\partial r}\left\langle e^{-i n q \theta} \phi_{n}\right\rangle_{b} \simeq \frac{1}{\Delta}\left\langle e^{-i n q \theta} \phi_{n}\right\rangle_{b}, I_{e}^{n l}$ can be evaluated in the same way. The result is

$$
I_{e}^{n l} \simeq-\left(\frac{\epsilon}{2}\right)^{\frac{1}{2}} \int_{0}^{1} d \kappa\left[k_{\theta}^{2} D_{\theta \theta}^{e}+\left(\frac{2 \kappa}{\Delta}\right)^{2} D_{r r}^{e}\right] \Delta \ln \left(\frac{2 \kappa x_{t}}{\Delta}\right) \hat{\phi}_{n}^{2}
$$

For $I_{i}^{n l}$, we note that the spectrum dependent quantities $D_{r r}^{i}$ and $D_{\theta \theta}^{i}$ varies slowly with $x$ on the spatial scale of $x_{i}$, because they involve $\left|\underset{\substack{m^{\prime} \\ n^{\prime}}}{0}\right|^{2}$ rather than $\phi_{\substack{m^{\prime} \\ n^{\prime}}}^{0}$. Therefore, in evaluating the integral $I_{i}^{n l}, D_{r r}^{i}(x)$ and $D_{\theta \theta}^{i}(x)$ can be replaced by $D_{r r}^{i}(0)$ and $D_{\theta \theta}^{i}(0)$. The integral $I_{i}^{n l}$ can then be easily evaluated,

$$
I_{i}^{n l}=\left[k_{\theta}^{2} D_{\theta \theta}^{i(0)}-\frac{i}{2} \mu D_{r r}^{i(0)}\right] \sqrt{\frac{\pi}{i \mu}} \hat{\phi}_{n}^{2}
$$


Noting Eq.(19), we multiply Eq.(43) on both side by $\sqrt{\frac{i \mu}{\pi}}$. Replacing $\hat{\phi}_{n}^{2}$ by $\left|\hat{\phi}_{n}^{m}\right|^{2}$, and then taking the imaginary part of the equation, we obtain,

$$
\begin{aligned}
\operatorname{Im\varepsilon }(\vec{k}, \omega)\left|\hat{\phi}_{n}^{m}\right|^{2} & =\left(\frac{\epsilon}{2}\right)^{\frac{1}{2}}\left(1-\frac{\omega_{e}^{*}}{\omega}\right) \Delta \ln \frac{x_{t}}{\Delta}\left|\hat{\phi}_{n}^{m}\right|^{2} \operatorname{Im}\left(g_{n} \sqrt{\frac{i \mu}{\pi}}\right) \\
& -\left(\frac{\epsilon}{2}\right)^{\frac{1}{2}} \int_{0}^{1} d \kappa\left[k_{\theta}^{2} \operatorname{Im}\left(D_{\theta \theta}^{e} \sqrt{\frac{i \mu}{\pi}}\right)+\left(\frac{2 \kappa}{\Delta}\right)^{2} \operatorname{Im}\left(D_{r r}^{e} \sqrt{\frac{i \mu}{\pi}}\right)\right] \Delta \ln \left(\frac{2 \kappa x_{t}}{\Delta}\right)\left|\hat{\phi}_{n}^{m}\right|^{2} \\
& +\left[k_{\theta}^{2} \operatorname{Im}\left(D_{\theta \theta}^{i(0)}\right)-\frac{1}{2} \mu \operatorname{Re}\left(D_{r r}^{i(0)}\right)\right] \mid \underset{n}{\left.\hat{\phi}_{n}\right|^{2}}
\end{aligned}
$$

For the same reasons as we have discussed in Sec.III the nonresonant terms associated with $\operatorname{Re}\left(g_{n}\right), \operatorname{Re}\left(D_{\theta \theta}^{e}\right), \operatorname{Re}\left(D_{r r}^{e}\right)$ and $\operatorname{Re}\left(D_{r r}^{i}\right)$ on the right side of the above equation can be neglected. The further reason for neglecting $R_{e} D_{r r}^{i}$ is that $k_{\theta}^{2}>\mu$ for the most unstable mode. By noting that $\operatorname{Im} \varepsilon(\vec{k}, \omega) \approx \frac{\partial e}{\partial \omega}\left(\gamma-\gamma^{\text {sd }}\right)$, and replacing $\gamma$ by $\frac{1}{2} \frac{\partial}{\partial t}$, we obtain the wave kinetic equation,

$$
\frac{\partial}{\partial t}\left|\phi_{n}\right|^{2}=2\left(\gamma_{e}^{l}+\gamma^{s d}+\gamma_{e}^{n l}+\gamma_{i}^{n l}\right)\left|\phi_{n}^{m}\right|^{2}
$$

In the above equation, $\gamma_{e}^{l}$ and $\gamma^{s d}$ are the linear growth rate and the shear damping rate given by Eq.(24) and Eq.(17), respectively, $\gamma_{i}^{n l}$ and $\gamma_{e}^{n l}$ are the nonlinear transfer rates due to ion Compton scattering and trapped electron Compton scattering. Using Eq.(47) and $\frac{\partial \epsilon}{\partial \omega}=-\frac{\omega}{\omega^{*}}$, we have,

$$
\begin{aligned}
& \frac{\gamma_{i}^{n l}}{\left|\omega_{\vec{k}}\right|}=-\frac{\omega_{\vec{k}}}{\left|\omega_{e}^{*}\right|} k_{\theta}^{2} \operatorname{Im} D_{\theta \theta}^{i} \\
& \frac{\gamma_{e}^{n l}}{\left|\omega_{\vec{k}}\right|}=\frac{1}{2} \frac{\omega_{\vec{k}}}{\left|\omega_{e}^{*}\right|}\left(\frac{\epsilon}{\pi}\right)^{\frac{1}{2}} \int_{0}^{1} d \kappa \frac{\Delta}{x_{t}} \ln \left(\frac{2 \kappa x_{t}}{\Delta}\right)\left[k_{\theta}^{2} \operatorname{Im} D_{\theta \theta}^{e}+\left(\frac{2 \kappa}{\Delta}\right)^{2} \operatorname{Im} D_{r \mathrm{r}}^{e}\right]
\end{aligned}
$$

where $D_{\theta \theta}^{i}$ is evaluated at $x=0$.

\section{Nonlinear Wave-Particle Interaction in a Sheared Magnetic Field}

In this section, nonlinear wave-particle interaction, namely, ion Compton scattering and trapped electron Compton scattering, in a sheared magnetic field are discussed. The effect of slab-like eigenmode structure on the nonlinear wave-particle interaction is 
explored. Specifically, we find that ion Compton scattering is enhanced while trapped electron Compton scattering is weakened. Therefore, ion Compton scattering rather than trapped electron Compton scattering is the dominant nonlinear saturation mechanism of trapped electron mode turbulence in a sheared magnetic field.

From Eqs.(49) and (50) and noting Eqs.(37a), (41a), and (41b), the nonlinear transfer rates due to ion Compton scattering and trapped electron Compton scattering can be rewritten as

$$
\begin{aligned}
\gamma_{i}^{n l} & =\frac{\omega_{\bar{k}}}{\omega_{e}^{*}} k_{\theta}^{2}\left(\frac{c}{B}\right)^{2} \sum_{\substack{l m^{\prime} \\
n^{\prime}}}\left|\frac{\partial}{\partial r} \phi_{\substack{m^{\prime} \\
n^{\prime}}}\right|^{2}\left(k_{\theta}^{\prime 2}-k_{\theta}^{2}\right) \rho_{s}^{2} I m G_{m n, m^{\prime} n^{\prime}}^{i} \\
\gamma_{e}^{n l} & =\frac{\omega_{\vec{k}}}{\omega_{e}^{*}}\left(\frac{\epsilon}{\pi}\right)^{\frac{1}{2}}\left(\frac{c}{B}\right)^{2} \int_{0}^{1} d \kappa \frac{\Delta}{x_{t}} \ln \left(\frac{x_{t}}{\Delta}\right) \sum_{n^{\prime}}\left(k_{\theta}^{\prime 2}-k_{\theta}^{2}\right) \rho_{s}^{2} \\
& \times\left\{k_{\theta}^{2}\left|\frac{\partial}{\partial r}\left\langle e^{-i n^{\prime} q \theta} \phi_{n^{\prime}}\right\rangle_{b}\right|^{2}+\left(\frac{2 \kappa}{\triangle}\right)^{2}\left|\left\langle e^{-i n^{\prime} q \theta} \phi_{n^{\prime}}\right\rangle_{b}\right|^{2}\right\} I m G_{n, n^{\prime}}^{e}
\end{aligned}
$$

From Eq.(38), the interaction kernel for ion Compton scattering is given by

$$
I m G_{m m^{\prime}, n n^{\prime}}^{i}=\left\langle J_{0}^{2}\left(k_{\perp} \rho_{i}\right) J_{0}^{2}\left(k_{\perp}^{\prime} \rho_{i}\right)\right\rangle_{\perp} \frac{\sqrt{\pi}}{\left|k_{\|}^{\prime} v_{i}\right|} e^{-\left(\frac{\psi^{\prime \prime}}{k_{\|}^{\prime \prime} \|_{i}}\right)^{2}}
$$

For trapped electron Compton scattering, we note that $\omega \gg \omega_{d e}$ and $\omega^{\prime} \gg \omega_{d e}^{\prime}$, so that $G_{n, n^{\prime}}^{e}$ in Eq.(31b) can be approximated by $G_{n, n^{\prime}}^{e} \approx-\frac{1}{2 \omega^{\prime \prime}}$, hence,

$$
\operatorname{Im} G_{n, n^{\prime}}^{e}=\frac{\pi}{2} \delta\left(\omega+\omega^{\prime}\right)
$$

Now, we discuss the physical implications of Eqs.(51)-(54). First, from the direct observation of Eqs.(51)-(54), we note that for $k_{\theta}<k_{\theta}^{\prime}, \gamma_{i}^{n l}>0$ but $\gamma_{e}^{n l}<0$, i.e. a long wavelength mode is nonlinearly excited by ion Compton scattering but nonlinearly damped by trapped electron Compton scattering; for $k_{\theta}>k_{\theta}^{\prime}, \gamma_{i}^{n l}<0$ but $\gamma_{e}^{n l}>0$, alternatively a short wavelength mode is nonlinearly damped by ion Compton scattering but nonlinearly excited by trapped electron Compton scattering. Therefore, we have two competitive nonlinear transfer processes: ion Compton scattering transfers wave energy from short to long wavelengths while trapped electron Compton scattering transfers wave 
energy from long to short wavelengths. Second, we note that the interaction kernel for ion Compton scattering has a broad band spectrum with width $\Delta \omega^{\prime \prime} \simeq k_{\|}^{\prime}(x) v_{i}$. Since the slab-like eigenmode spectrum $\left|\phi_{n}\right|^{2}$ can extend to $x_{i}$, the width of the interaction kernel is $\Delta \omega^{\prime \prime} \sim k_{\|}^{\prime}\left(x_{i}^{\prime}\right) v_{i} \sim \omega^{\prime}$, which indicates any beat mode with frequency $\omega^{\prime \prime}$ in the range $\Delta \omega^{\prime \prime} \sim \omega^{\prime}$ can be very easily coupled with the ions. T'he ion Compton scattering process is therefore considerably enhanced. This frequency spread or "resonance broadening" is caused by the magnetic shear induced Doppler shift. In order to see this, it is instructive to note that in the shearless case, we have $\omega^{\prime} \gg k_{\|}^{\prime} v_{i}$. Thus the interaction kernel in the shearless case can be obtained by taking the limit $k_{\|}^{\prime} v_{i} \rightarrow 0$,

$$
\lim _{k_{\|}^{\prime} v_{i} \rightarrow 0} I m G_{m m^{\prime}, n n^{\prime}}^{i}=\left\langle J_{0}^{2}\left(k_{\perp} \rho_{i}\right) J_{0}^{2}\left(k_{\perp}^{\prime} \rho_{i}\right)\right\rangle_{\perp} \pi \delta\left(\omega^{\prime \prime}\right)
$$

namely, the ion Compton scattering is (severely) restricted to be local in frequency. As a consequenci of the Doppler 'resonance broadening', the ion Compton scattering process in a sheared magnetic field is fundamentally different from that in a shearless case. This is because: 1 ) local energy transfer (with comparable wave numbers $k_{\theta}^{\prime} \sim k_{\theta}$ ) which is absent in the shearless case is now very robust in the long wavelength part of the spectrum. ii.) ion Compton scattering is no longer an adiabatic process, namely, significant amount of wave energy is transfered directly to the ions (nonlinear ion heating). Third, due to the radial localization of trapped electron response, trapped electron Compton scattering is restricted to a very narrow layer of width $\Delta$ near mode rational surface. Hence, the intensity of the scattering is significantly reduced. This is apparent from Eq.(52) that $\gamma_{e}^{n l} \propto \frac{\Delta}{x_{t}} \ln \frac{x_{t}}{\Delta}$ and the fact that $\left|\left\langle e^{-i n^{\prime} q \theta} \phi_{n^{\prime}}\right\rangle_{b}\right|^{2}$ is much smaller than $\left|\phi_{n^{\prime}}\right|^{2}$.

We now evaluate the nonlinear couplings (i.e., the summations) in Eq.(51) and (52). Since the linear modes are densely packed, we can treat both ' $m^{\prime}$ and $n^{\prime}$ as continuous variables, so that the summations in both Eq.(51) and Eq.(52) can be converted into integrals according to $\sum_{\substack{m_{n^{\prime}}^{\prime} \\ n^{\prime}}}=\int d n^{\prime} \int d m^{\prime}$. Noting that $k_{\theta}^{\prime}=\frac{n^{\prime} q}{r}$ and $m^{\prime}=n^{\prime} q\left(r_{\substack{m^{\prime} \\ n^{\prime}}}\right)$, we 
have $d n^{\prime}=\frac{r}{q} d k_{\theta}^{\prime}$, and $d m^{\prime}=\frac{1}{\Delta^{\prime}} d r_{n^{\prime}} \underset{\text { for fixed }}{ } n^{\prime}$, hence,

$$
\sum_{\substack{m^{\prime} \\ n^{\prime}}}=\frac{r}{q} \int \frac{d k_{\theta}^{\prime}}{\Delta^{\prime}} \int d r_{\substack{m_{n^{\prime}}^{\prime} \\ n^{\prime}}}
$$

In order to complete the integral, an explicit form of the spatial structure of the spectrum function $\left|\phi_{n}\right|^{2}$ is requred. The integral is only sensitive to the spatial width of the spectrum $w^{\prime}$ rather than to the exact form of its spatial structure. By noting that $\left|\phi_{n}^{m}\right|^{2}$ changes slowly for $x^{\prime}<w^{\prime}$, but decays rapidly for $x^{\prime}>w^{\prime}$, we can approximate $\left|\phi_{n}^{m}\right|^{2}$ as

$$
\left|\phi_{n}^{m}\right|^{2}=\left|\phi\left(k_{\theta}\right)\right|^{2} \theta\left(\left|x^{\prime}\right|-w^{\prime}\right)
$$

where $\theta\left(\left|x^{\prime}\right|-w^{\prime}\right)$ is the heavyside step function.

\section{a. Nonlinear Transfer Rate Due to Ion Compton Scattering}

In evaluating $\gamma_{i}^{n !}$, noting that in the limit $T_{e} \gg T_{i},\left\langle J_{0}^{2}\left(k_{\perp} \rho_{i}\right) J_{0}^{2}\left(k_{\perp}^{\prime} \rho_{i}\right)\right\rangle_{\perp}$ can be approximated $b_{j}$ 1. From Eqs.(51), and (53), and noting that $\left|\frac{\partial}{\partial r} \phi_{m_{n^{\prime}}}\right|^{2} \approx \mu^{\prime 2} x^{\prime 2}|\phi|^{2}\left(k_{\theta}^{\prime}\right) \theta\left(\left|x^{\prime}\right|-\right.$ $\left.w^{\prime}\right), k_{\|}^{\prime} v_{i}=\frac{\omega^{\prime} x^{\prime}}{x_{i}^{\prime}}, x_{i}^{\prime}=\frac{\omega^{\prime} L_{l}}{k_{i}^{\prime} v_{i}}$, the nonlinear transfer rate for ion Compton scattering can be rewritten as

$$
\begin{aligned}
\frac{\gamma_{i}^{n l}}{|\omega|} & =\left(\frac{c}{B}\right)^{2} \frac{k_{\theta}^{2}}{\left|\omega_{e}^{*}\right|} \frac{r}{q} \int \frac{d k_{\theta}^{\prime}}{\Delta^{\prime}}|\phi|^{2}\left(k_{\theta}^{\prime}\right)\left(k_{\theta}^{\prime 2}-k_{\theta}^{2}\right) \rho_{\theta}^{2} \\
& \times \int_{r-\omega^{\prime}}^{r+\omega^{\prime}} d r_{n_{n^{\prime}}^{\prime}} \mu^{\prime 2} x^{\prime 2} \frac{\sqrt{\pi} x_{i}^{\prime}}{\left|\omega^{\prime} x^{\prime}\right|} \exp \left\{\cdots\left(\frac{\omega^{\prime \prime} x_{i}^{\prime}}{\omega^{\prime} x^{\prime}}\right)^{2}\right\}
\end{aligned}
$$

It is imporiant to note that in a sheared magnetic field the radial wavenumber $k_{r}^{\prime}$ is a function of $x^{\prime}$, i.e., $k_{r}^{\prime}=-\mu^{\prime} x^{\prime}$, which implies that nonlinear ion coupling is stronger for larger $x^{\prime}$. The fact that the slab-like eigenrnode spectrum extends to $x_{i}^{\prime}$ renders $\left|k_{r}^{\prime}\right| \sim$ $\left|\mu^{\prime} x_{i}^{\prime}\right| \sim \sqrt{\frac{L_{1}}{L_{n}}} x_{t}^{\prime-1}>x_{t}^{\prime-1}$. Hence, due to the strong noninear coupling, ion Compton scattering is expected to be much more robust in a sheared magnetic field. Thus, sat ration at levels below the conventionally quoted mixing length estimate of $\frac{\tilde{n}}{n_{0}} \sim \frac{x_{1}}{L_{n}}$ appears likely. Note also that since $k_{r}^{\prime} \rho_{i} \leq 1$, the replacing of $\left\langle J_{0}^{2}\left(k_{\perp} \rho_{i}\right) J_{0}^{2}\left(k_{\perp}^{\prime} \rho_{i}\right)\right\rangle_{\perp}$ by 1 in the weak 
turbulence theory constitutes only a slight quantitative error. Changing variable from $r_{n^{\prime}}$ to $x^{\prime}=r-r_{\substack{m^{\prime} \\ n^{\prime}}}$ and carrying out the $x^{\prime}$ integration in the above equation, we have

$$
\frac{\gamma_{i}^{n l}}{|\omega|}=\sqrt{\pi} \frac{T_{e}}{T_{i}}\left(\frac{c}{B}\right)^{2} \frac{k_{\theta}^{2}}{\left|\omega_{e}^{\prime}\right|} \frac{r}{q} \int \frac{d k_{\theta}^{\prime}}{\left|\omega^{\prime}\right|}|\phi|^{2}\left(k_{\theta}^{\prime}\right)\left(k_{\theta}^{\prime 2}-k_{\theta}^{2}\right) \frac{w^{\prime}}{x_{i}^{\prime}} \frac{2 w^{\prime}}{\Delta^{\prime}} E_{2}\left[\left(\frac{\omega^{\prime \prime} x_{i}^{\prime}}{\omega^{\prime} w^{\prime}}\right)^{2}\right]
$$

where $E_{2}(x)=\int_{1}^{\infty} d y y^{-2} \exp \{-x y\}$ is the exponential function. $E_{2}\left[\left(\frac{\omega^{\prime \prime} x_{i}^{\prime}}{\omega^{\prime} w^{\prime}}\right)^{2}\right]$ is sharply peaked around $\omega^{\prime \prime}$ with a frequency spread $\Delta \omega^{\prime \prime} \sim \omega^{\prime}\left(\frac{w^{\prime}}{x_{i}^{\prime}}\right)$. Noting that

$$
\frac{\omega^{\prime \prime}}{\omega^{\prime}}=\frac{\left(k_{\theta}^{\prime}-k_{\theta}\right)\left(1-k_{\theta} k_{\theta}^{\prime} \rho_{s}^{2}\right)}{k_{\theta}^{\prime}\left(1+k_{\theta}^{2}\right)\left(1+k_{\theta}^{\prime \prime}\right)}
$$

small $\omega^{\prime \prime}$ can be achieved either around $k_{\theta}^{\prime} \sim k_{\theta}^{+}=k_{\theta}$ (local interaction) or around $k_{\theta}^{\prime} \sim$ $k_{\theta}^{-}=\frac{1}{k_{\theta} \rho_{\theta}^{2}}$ (nonlocal interaction) which is depicted in Fig.2. The fact that $E_{2}\left[\left(\frac{\omega^{\prime \prime} x_{i}^{\prime}}{\omega^{\prime} \omega^{\prime}}\right)^{2}\right]$ is sharply peaked around $k_{\theta}^{+}$and $k_{\theta}^{-}$allows us to simplify the expression for $\gamma_{i}^{n l}$ considerably, namely, to convert the integral operator in Eq.(58) into a differential operator. In order to do this, let's expand $|\phi|^{2}\left(k_{\theta}^{\prime}\right)$ in a Taylor series around $k_{\theta}^{j}=k_{\theta}^{+}, k_{\theta}^{-}$, i.e.

$$
|\phi|^{2}\left(k_{\theta}^{\prime}\right)=|\phi|^{2}\left(k_{\theta}^{j}\right)+\frac{\partial}{\partial k_{\theta}^{\prime}}|\phi|^{2}\left(k_{\theta}^{j}\right)\left(k_{\theta}^{\prime}-k_{\theta}^{j}\right)+\cdots
$$

This expansion is valid only when

$$
\frac{\partial}{\partial k_{\theta}}|\phi|^{2}\left(k_{\theta}^{j}\right)\left(k_{\theta}^{\prime}-k_{\theta}^{j}\right)<|\phi|^{2}\left(k_{\theta}^{j}\right)
$$

Taking $k_{\theta}^{\prime}-k_{\theta}^{j} \sim \overline{\Delta k_{\theta} E_{2}}$ and $\frac{1}{\mid \phi^{2}\left(k_{0}^{j}\right)} \frac{\partial}{\partial k_{0}}|\phi|^{2}\left(k_{\theta}^{j}\right)=\frac{1}{\Delta k_{0}}$, where $\overline{\Delta k_{\theta}} E_{2}$ is the spectrum width of the interaction kernel $E_{2}\left[\left(\frac{\omega^{\prime \prime} x_{i}^{\prime}}{\omega^{\prime} w^{\prime}}\right)^{2}\right]$, and $\overline{\Delta k_{\theta_{\phi}}}$ is the spectrum width of $|\phi|^{2}\left(k_{\theta}\right)$, the condition above can be rewriten as

$$
{\overline{\Delta k_{\theta} E_{2}}}<{\overline{\Delta k_{\theta \phi}}}
$$

namely, the spectrum width of the interaction kernel must be narrower than the width of the fluctuation spectrum.

To simplify Eq.(58), we first calculate the local interaction contribution to $\gamma_{i}^{\text {nl }}$. We define a new variable $k_{\theta}^{\prime \prime}=k_{\theta}^{\prime}-k_{\theta}$. Near $k_{\theta}$, we have $\frac{\omega^{\prime \prime} x_{i}^{\prime}}{\omega^{\prime} w^{\prime}} \simeq \alpha^{+} k_{\theta}^{\prime \prime}$, where $\alpha^{+}=$ 
$\left|\frac{1-k_{\theta}^{2} \rho_{\rho}^{2}}{k_{\theta}\left(1+k_{\theta}^{2} \rho^{2}\right)}\right| \frac{x_{i}^{\prime}}{w^{\prime}} \equiv \frac{1}{\Delta k_{\theta} E_{2}}$. Noting that $k_{\theta}^{\prime 2}-k_{\theta}^{2}=k_{\theta}^{\prime \prime 2}+2 k_{\theta} k_{\theta}^{\prime \prime}$ and making use of Eq.(59), we have

$$
\begin{aligned}
& \int_{\text {neark+ }} \frac{d k_{\theta}}{\left|\omega^{\prime}\right|}|\phi|^{2}\left(k_{\theta}^{\prime}\right)\left(k_{\theta}^{\prime 2}-k_{\theta}^{2}\right) \frac{w^{\prime}}{x_{i}^{\prime}} \frac{2 w^{\prime}}{\Delta^{\prime}} E_{2}\left[\left(\frac{\omega^{\prime \prime} x_{i}^{\prime}}{\omega^{\prime} w^{\prime}}\right)^{2}\right] \\
= & \frac{1}{|\omega|} \frac{w}{x_{i}} \frac{2 w}{\Delta} \int_{-\infty}^{\infty} d k_{\theta}^{\prime \prime}\left(k_{\theta}^{\prime \prime 2}+2 k_{\theta} k_{\theta}^{\prime \prime}\right)\left[|\phi|^{2}\left(k_{\theta}\right)+\frac{\partial}{\partial k_{\theta}}|\phi|^{2}\left(k_{\theta}\right) k_{\theta}^{\prime \prime}+\cdots\right] E_{2}\left(\alpha^{+^{2}} k_{\theta}^{\prime \prime 2}\right) \\
\approx & \frac{\sqrt{\pi}}{5} \frac{1}{|\omega|} \frac{w}{x_{i}} \frac{2 w}{\Delta} \frac{1}{\alpha^{+3}}\left\{|\phi|^{2}\left(k_{\theta}\right)+2 k_{\theta} \frac{\partial}{\partial k_{\theta}}|\phi|^{2}\right\}
\end{aligned}
$$

In the above calculation, $\int_{-\infty}^{\infty} d k_{\theta}^{\prime \prime} k_{\theta}^{\prime \prime 2} E_{2}\left(\alpha^{+^{2}} k_{\theta}^{\prime \prime 2}\right)=\frac{\sqrt{\pi}}{5} \frac{1}{\alpha^{+3}}$ has been used, and higher order terms in $\frac{\overline{\Delta k_{\theta} E_{2}}}{\Delta k_{0}}$ have been neglected.

Next, we calculate the nonlocal interaction contribution to $\gamma_{i}^{n l}$. We define $k_{\theta}^{\prime \prime}=$ $k_{\theta}^{\prime}-k_{\theta}^{-}$. Near $k_{\theta}^{-}$, we have $\left(\frac{\omega^{\prime \prime} x_{i}^{\prime}}{\omega^{\prime} w^{\prime}}\right)^{2} \simeq \alpha^{-2} k_{\theta}^{\prime \prime 2}$, where $\alpha^{-}=k_{\theta}^{2} \rho_{s}^{2}\left|\frac{1-k_{\theta}^{2} \rho_{c}^{2}}{k_{\theta}\left(1+k_{\theta}^{2} \rho_{\theta}^{2}\right)}\right| \frac{x_{i}^{\prime}}{w^{\prime}}$. Noting that $k_{\theta}^{\prime 2}-k_{\theta}^{2}=\left(k_{\theta}^{-2}-k_{\theta}^{2}\right)+2 k_{\theta}^{-} k_{\theta}^{\prime \prime}+k_{\theta}^{\prime \prime 2}$, and $\left.\frac{1}{\left|\omega^{\prime}\right|} \frac{w^{\prime}}{x_{i}^{\prime}} \frac{w^{\prime}}{\Delta^{\prime}}\right|_{k_{\theta}^{-}}=\frac{1}{|\omega|} \frac{w}{x_{i}} \frac{w}{\Delta}$, we have

$$
\begin{aligned}
& \int_{\text {nearke }} \frac{d k_{\theta}}{\left|\omega^{\prime}\right|}|\phi|^{2}\left(k_{\theta}^{\prime}\right)\left(k_{\theta}^{\prime 2}-k_{\theta}^{2}\right) \frac{w^{\prime}}{x_{i}^{\prime}} \frac{2 w^{\prime}}{\Delta^{\prime}} E_{2}\left[\left(\frac{\omega^{\prime \prime} x_{i}^{\prime}}{\omega^{\prime} w^{\prime}}\right)^{2}\right] \\
= & \frac{1}{|\omega|} \frac{w}{x_{i}} \frac{2 w}{\Delta} \int_{-\infty}^{\infty} d k_{\theta}^{\prime \prime}\left[\left(k_{\theta}^{-2}-k_{\theta}^{2}\right)+2 k_{\theta} k_{\theta}^{\prime \prime}+k_{\theta}^{\prime \prime 2}\right] \\
& \left.\times\left.|| \phi\right|^{2}\left(k_{\theta}^{-}\right)+\frac{\partial}{\partial k_{\theta}}|\phi|^{2}\left(k_{\theta}^{-}\right) k_{\theta}^{\prime \prime}+\cdots\right] E_{2}\left(\alpha^{-2} k_{\theta}^{\prime \prime 2}\right) \\
\approx & \frac{1}{|\omega|} \frac{w}{x_{i}} \frac{2 w}{\Delta}\left(k_{\theta}^{-2}-k_{\theta}^{2}\right)|\phi|^{2}\left(k_{\theta}^{-}\right) \frac{2 \sqrt{\pi}}{3} \frac{1}{\alpha^{-}},
\end{aligned}
$$

In the above calculation, $\int_{-\infty}^{\infty} d k_{\theta}^{\prime \prime} E_{2}\left(\alpha^{-2} k_{\theta}^{\prime \prime 2}\right)=\frac{2 \sqrt{\pi}}{3} \frac{1}{\alpha^{-}}$has been used, and high order terms has been neglected as before. Now, combining Eq.(60), and Eq.(61), we obtain the nonlinear tranfer rate for ion Compton scattering,

$$
\begin{aligned}
& \frac{\gamma_{i}^{n l}}{|\omega|}=\frac{4 \pi}{5} \frac{\omega}{\omega_{e}^{*}} \frac{T_{e}}{T_{i}}\left(\frac{c}{B}\right)^{2}\left(\frac{k_{\theta}}{\omega}\right)^{2} \frac{r}{q} \frac{w}{x_{i}} \frac{w}{\Delta} \\
& \times\left\{\frac{1}{\alpha^{+3}} \sqrt{k_{\theta}} \frac{\partial}{\partial k_{\theta}}\left[\sqrt{k_{\theta}}|\phi|^{2}\left(k_{\theta}\right)\right]+\frac{5}{3} \frac{1}{\alpha^{-}}\left(k_{\theta}^{-2}-k_{\theta}^{2}\right)|\phi|^{2}\left(k_{\theta}^{-}\right)\right\}
\end{aligned}
$$

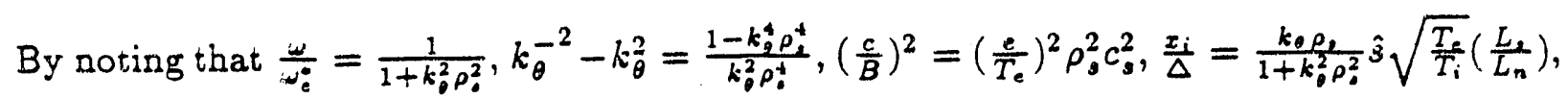
the above equation can be rewritten as

$$
\frac{\gamma_{i}^{n l}}{|\omega|}=\frac{4 \pi}{5}\left(\frac{r \hat{s}}{q \rho_{s}}\right)\left(\frac{\Omega_{i}}{\omega}\right)^{2}\left(\frac{T_{e}}{T_{i}}\right)^{\frac{3}{2}}\left(\frac{L_{s}}{L_{n}}\right)\left(\frac{w}{x_{i}}\right)^{3}\left(\frac{e}{T_{e}}\right)^{2}
$$




$$
\times\left\{\left(\frac{w}{x_{i}}\right)^{2} \frac{\left(k_{\theta} \rho_{s}\right)^{6}\left(1+k_{\theta}^{2} \rho_{s}^{2}\right)}{\left|1-k_{\theta}^{2} \rho_{s}^{2}\right|^{3}} \sqrt{k_{\theta}} \frac{\partial}{\partial k_{\theta}}\left[\sqrt{k_{\theta}}|\phi|^{2}\left(k_{\theta}\right)\right]+\frac{5}{3} \operatorname{sign}\left(1-k_{\theta}^{2} \rho_{s}^{2}\right)|\phi|^{2}\left(k_{\theta}^{-}\right)\right\} .
$$

In the above equation, the first term on the right side is the local transfer rate and corresponds to a spectral flow in $k_{\theta}$ space. The second term is the nonlocal transfer rate, which is positive for $k_{\theta} \rho_{s}<1$, and negtive for $k_{\theta} \rho_{s}>1$.

\section{b. Nonlinear Transfer Rate Due to Trapped Electron Compton Scattering}

In order to evaluate $\gamma_{e}^{n l}$, let's first calculate the quantity $I_{n^{\prime}}=\left|\left\langle e^{-i n^{\prime} q \theta} \phi_{n^{\prime}}\right\rangle_{b}\right|^{2}$. By using Fourier representation in $\theta$ for $\phi_{n^{\prime}}$, i.e. $\phi_{n^{\prime}}=\sum_{m^{\prime}} \phi_{n_{n^{\prime}}} e^{i m^{\prime} \theta}$, we can write

$$
I_{n^{\prime}}=\sum_{m^{\prime}} \sum_{p=-\infty}^{\infty} C_{m^{\prime}, m^{\prime}+p}^{n^{\prime}} \phi_{\substack{m^{\prime} \\ n^{\prime}}} \phi_{m^{\prime}+p}^{*},
$$

where the coupling coefficient between different mode rational surface for fixed $n^{\prime}$ is $C_{m^{\prime}, m^{\prime}+p}^{n^{\prime}}=\left\langle e^{i\left(m^{\prime}-n^{\prime} q\right) \theta}\right\rangle_{b}\left\langle e^{-i\left(m^{\prime}+p-n^{\prime} q\right) \theta}\right\rangle_{b}$. Since $\left\langle e^{i\left(m^{\prime}-n^{\prime} q\right) \theta}\right\rangle_{b}$ is localized around a mode rational surface $r_{\substack{m^{\prime} \\ n^{\prime}}}$ with spatial width $\Delta^{\prime}, C_{m^{\prime}, m^{\prime}+p}^{n^{\prime}}$ which is centered at $p=0$ will be a decaying and oscillatory function of $p$. Therefore, the primary contribution to $I_{n^{\prime}}$ comes from the $p=0$ term in Eq.(64). As a matter of fact, it has been shown in Ref.(2) that by keeping all $\mathrm{p}$ terms in Eq.(64), $I_{n^{\prime}}$ is only a factor of $\frac{\pi}{2}$ times that obtained by keepirg the $p=0$ term only. Hence, we may approximate $I_{n^{\prime}}$ as

$$
I_{n^{\prime}}=\frac{\pi}{2} \sum_{m^{\prime}}\left|\phi_{\substack{m^{\prime} \\ n^{\prime}}}\right|^{2}\left|\left\langle e^{i\left(m^{\prime}-n^{\prime} q\right) \theta}\right\rangle_{b}\right|^{2}
$$

The quantity $\left\langle e^{i\left(m^{\prime}-n^{\prime} q\right) \theta}\right\rangle_{6}$ can be calculated analytically for $\kappa \ll 1$, since Eq.(52) involves only an integral over $\left|\left\langle e^{i\left(m^{\prime}-n^{\prime} q\right) \theta}\right\rangle_{b}\right|^{2}$, the answer is not expected to be sensitive to this approximation. Employing $m^{\prime}-n^{\prime} q(r) \simeq \frac{x^{\prime}}{\Delta^{\prime}}$ yields

$$
\left\langle e^{i\left(m^{\prime}-n^{\prime} q\right) \theta}\right\rangle_{b} \simeq J_{0}\left(2 \kappa \frac{x^{\prime}}{\Delta^{\prime}}\right)
$$

Substituting Eqs.(55), (56), and (66) into Eq.(64), and carrying out the $x^{\prime}$ integration by noting the relation that $\int_{0}^{t_{0}} d t J_{0}^{2}(t) \approx \frac{1}{\pi} \ln t_{0}$ for $t_{0} \gg 1$, yields

$$
\begin{aligned}
I_{n^{\prime}} & =\frac{\pi}{2} \int_{-w^{\prime}}^{w^{\prime}} \frac{d x^{\prime}}{\Delta^{\prime}} J_{0}^{2}\left(2 \kappa \frac{x^{\prime}}{\Delta^{\prime}}\right)|\phi|^{2}\left(k_{\theta}^{\prime}\right) \\
& =\frac{1}{2 \kappa} \ln \left(\frac{2 \kappa w^{\prime}}{\Delta^{\prime}}\right)|\phi|^{2}\left(k_{\theta}^{\prime}\right) .
\end{aligned}
$$


Noting that

$$
\left|\frac{\partial}{\partial r}\left\langle e^{-i n^{\prime} q \theta} \phi_{n^{\prime}}\right\rangle_{b}\right|^{2} \approx\left(\frac{2 \kappa}{\Delta^{\prime}}\right)^{2}\left|\left\langle e^{-i n^{\prime} q \theta} \phi_{n^{\prime}}\right\rangle_{b}\right|^{2},
$$

and carrying out the $\kappa$ integration in Eq.(52), we can rewrite the nonlinear transfer rate for trapped electron Compton scattering as:

$$
\frac{\gamma_{e}^{n l}}{|\omega|} \approx \frac{\sqrt{\pi \epsilon}}{|| \omega \mid} \frac{\omega}{\omega_{e}^{*}}\left(\frac{c}{B}\right)^{2} \hat{s}^{2} k_{\theta}^{2} \rho_{s}^{2} \frac{\Delta}{x_{t}} \ln \frac{x_{t}}{\Delta} \sum_{n^{\prime}} k_{\theta}^{\prime 2}|\phi|^{2}\left(k_{\theta}^{\prime}\right)\left(k_{\theta}^{2}-k_{\theta}^{\prime 2}\right) \delta\left(\omega-\omega^{\prime}\right) \ln \frac{w^{\prime}}{\Delta^{\prime}}
$$

In arriving at Eq.(68), change of variable $k_{\theta}^{\prime} \rightarrow-k_{\theta}^{\prime}$ has been made. Now, converting the summation over $n^{\prime}$ in the above equation into an integral by noting that, $\sum_{n^{\prime}}=\frac{r}{q} \int d k_{\theta}^{\prime}$, and using the relation

$$
\delta\left(\omega_{n^{\prime}}-\omega_{n}\right)=\sum_{j=+,-} \frac{\delta\left(k_{\theta}^{\prime}-k_{\theta}^{j}\right)}{\left|\frac{\partial \omega^{\prime}}{\partial k_{\theta}^{\prime}}\right|}
$$

where $k_{\theta}^{+}=k_{\theta}$ and $k_{\theta}^{-}=\frac{1}{k_{\theta} \rho_{\theta}^{2}}$. we can further simplify the above equation to

$$
\begin{aligned}
\frac{\gamma_{e}^{n l}}{|\omega|} & =\sqrt{\pi \epsilon} \frac{\omega}{\omega_{e}^{*}}\left(\frac{c}{B}\right)^{2} \hat{s}^{2} \frac{k_{\theta}^{2} \rho_{s}^{2}}{|\omega|} \frac{\Delta}{x_{t}} \ln \frac{x_{t}}{\Delta} \\
& \times \frac{r}{q} \int d k_{\theta}^{\prime} k_{\theta}^{\prime 2}|\phi|^{2}\left(k_{\theta}^{\prime}\right)\left(k_{\theta}^{2}-k_{\theta}^{\prime 2}\right) \ln \frac{w^{\prime}}{\Delta^{\prime}} \sum_{j=+,-\left|\frac{\partial}{\partial k_{\theta}^{\prime}} \omega\left(k_{\theta}^{j}\right)\right|} \frac{\delta\left(k_{\theta}^{\prime}-k_{\theta}^{j}\right)}{\mid} \\
& =\sqrt{\pi \epsilon} \frac{\omega}{\omega_{e}^{*}}\left(\frac{\partial}{B}\right)^{2} \hat{s}^{2} \frac{k_{\theta}^{2} \rho_{s}^{2}}{|\omega|} \frac{\Delta}{x_{t}} \ln \frac{x_{t}}{\triangle} \\
& \times \frac{r}{q} k_{\theta}^{-2}|\phi|^{2}\left(k_{\theta}^{-}\right)\left(k_{\theta}^{2}-k_{\theta}^{-2}\right) \ln \frac{w}{\Delta} \frac{1}{\left|\frac{\partial}{\partial k_{\theta}} \omega\left(k_{\theta}^{--}\right)\right|}
\end{aligned}
$$

Replacing $k_{\theta}^{-}$with $\frac{1}{k_{\theta} \rho_{\theta}^{2}}$, and noting that $\left.\frac{\partial \omega}{\partial k_{\theta}}\right|_{k_{\theta}^{-}}=-\frac{c_{\theta} \rho_{0}}{L_{n}} \frac{1-k_{\theta}^{2} \rho_{\theta}^{2}}{1+k_{\theta}^{2} \rho_{\theta}^{2}}$, after a lengthy but straightforward algebraic manipulation, we obtain the final form for $\gamma_{e}^{n l}$,

$$
\begin{aligned}
\frac{\gamma_{e}^{n l}}{|\omega|} & =-\sqrt{\pi \epsilon} \hat{s}^{2}\left(\frac{r \hat{s}}{q \rho_{s}}\right)\left(\frac{\Omega_{i}}{\omega}\right)^{2}\left(\frac{T_{e}}{T_{i}}\right)^{\frac{1}{2}}\left(\frac{L_{s}}{L_{n}}\right)\left(\frac{w}{x_{i}}\right) \\
& \times\left(\frac{\triangle}{x_{t}}\right) \ln \left(\frac{x_{t}}{\triangle}\right)\left(\frac{\triangle}{w}\right) \ln \left(\frac{w}{\triangle}\right) \frac{\operatorname{sign}\left(1-k_{\theta}^{2} \rho_{s}^{2}\right)}{1+k_{\theta}^{2} \rho_{s}^{2}}\left(\frac{e}{T_{e}}\right)^{2}|\phi|^{2}\left(k_{\theta}^{-}\right)
\end{aligned}
$$

The above equation shows that trapped electron Compton scattering is a nonlocal transfer process. Local interactions $\left(k_{\theta}^{\prime}=k_{\theta}\right)$ do not contribute because $k_{\theta}^{\prime 2}-k_{\theta}^{2}=0$. 
For Pearlstein-Berk eigenmode structure, we have $w \sim x_{i}$. Comparing Eqs.(63) and (71), we find that the nonlinear transfer rate due to trapped electron Compion scattering is considerably smaller than that due to ion Compton scattering. This is because: i.) the spectrum of Pearlstein-Berk eigenmode extends to $x_{i}$ so that ion Compton scattering can take place in a frequency range $\omega+\omega^{\prime}<k_{\|}^{\prime}\left(x_{i}^{\prime}\right) v_{i} \sim \omega^{\prime}$. However, the trapped electron Compton scattering process is strictly limited by the $\delta\left(\omega^{\prime}-\omega\right)$ function. As a consequence, the local interaction, which is absent in trapped electron Compton scattering process, is very intense in ion Comptor scattering. ii.) trapped electron Compton scattering is radially restricted to a very narrow region of width $\Delta \ll x_{i}$ near mode rational surface while ion Compton scattering extesids to $x_{i}$. Due to this radial localization effect, the intensity of trapped electron Compion scattering is reduced considerably. This reduction is apparent from Eq.(T1), the reduction factor is approximately given by $\frac{\Delta}{x_{0}} \ln \left(\frac{x_{i}}{\Delta}\right) \frac{\Delta}{x_{i}} \ln \left(\frac{x_{i}}{\Delta}\right) \sim\left(\frac{L_{n}}{L_{0}}\right)^{\frac{3}{2}} \ll$ 1. Therefore, ion Compton scattering, rather than trapped electron Compton scattering is the dominant nonlinear saturation process for the slab-like trar ped electron mode.

\section{Saturated Fluctuation Spectrum}

In this section, the saturated fluctuation spectrum is calculated. The saturated state is defined by the condition $\frac{\partial}{\partial t}\left|\phi_{n}\right|^{2}=0$. From the wave kinetic equation, it implies

$$
\gamma_{e}^{l}+\gamma^{s d}+\gamma_{e}^{n l}+\gamma_{i}^{n l}=0
$$

namely, the linear growth $\gamma_{e}^{\prime}$ at each wavenumber $k_{\theta}$ is balanced by the combined effect of linear shear damping and the nonlinear transfer due to trapped esectron Compton scattering and ion Comptor scattering. The trapped electron Compton scattering, which has been shown in last section to be much weaker than the ion Compton scattering, is hereafter neglected. Shear damping can be neglected when we consider the most unstable mode. Consequently, satuaration is determined by the balance between the linear growth due to the trapped electron excitation and the nonlinear transfer due to the ion Compton scattering. Shear damping is only important when we consider a linearly marginal stable mode 
$\left(\gamma_{e}^{l} \leq \gamma^{s d}\right)$, which determines the spectral cutoff. Thus, by neglecting both $\gamma_{e}^{n l}$ and $\gamma^{\text {sd }}$ in Eq.(72), the saturation condition can be reduced to,

$$
\gamma_{i}^{n l, c}+\gamma_{i}^{n l, d}+\gamma_{e}^{l}=0
$$

where $\gamma_{i}^{n l, c}$ and $\gamma_{i}^{n l, d}$ are the nonlinear transfer rate due to the local and nonlocal interactions in ion Compton scattering, which corresponds to the first and second term on the right side of Eq.(63), respectively.

Now, let's solve Eq.(73) for fluctuation spectrum at saturation. In the region where $k_{\theta} \rho_{s}>1$, the nonlocal interaction is due to the beating of a high- $k_{\theta}$ and a low- $k_{\theta}^{\prime}$ fluctuation of similar frequency $\omega \sim \omega^{\prime}$. The intensity of the interaction is determined by the wave spectrum in region $k_{\theta} \rho_{s}<1$. Due to the nature of the ion Compton scattering process, we can anticipate that for even moderate long wavelength fluctuation levels, the nonlocal interaction is strong and causes a nonlinear transfer rate which exceeds the growth rate due to trapped electron excitation. As a consequence, these high- $k_{\theta}$ modes are only very feebly excited (if at all), and thus do not contribute significantly to either the fluctuation level or the transport. Thus, the spectrum is populated only for $k_{\theta} \rho_{s}<1$.

In region $k_{\theta} \rho_{\mathrm{s}}<1$, the nonlocal interaction is negligible. The remainder of $\mathrm{Eq}_{\mathrm{q}} .(73)$ is then a local first order differential equation and can be easily solved. In order to simplify the analysis, we introduce a new variable $\bar{k}_{\theta} \equiv k_{\theta} \rho_{\mathrm{g}}$. With $\bar{k}_{\theta}$, we can rewrite $\gamma_{i}^{n l, c}$ and $\gamma_{e}^{l}$ as

$$
\begin{aligned}
\frac{\gamma_{i}^{n l, \mathrm{c}}}{|\omega|} & =\frac{4 \pi}{5}\left(\frac{r \hat{3}}{q \rho_{s}}\right)\left(\frac{L_{n}}{\rho_{s}}\right)^{2}\left(\frac{T_{e}}{T_{i}}\right)^{\frac{3}{2}}\left(\frac{L_{s}}{L_{n}}\right)\left(\frac{w}{x_{i}}\right)^{5}\left(\frac{e}{T_{e}}\right)^{2} \bar{k}_{\theta}^{4} \sqrt{\bar{k}_{\theta}} \frac{\partial}{\partial \bar{k}_{\theta}}\left[\sqrt{\bar{k}_{\theta}}|\phi|^{2}\left(\bar{k}_{\theta}\right)\right] \\
& \equiv A_{i}^{n l} \bar{k}_{\theta}^{4} \sqrt{\bar{k}_{\theta}} \frac{\partial}{\partial \bar{k}_{\theta}}\left[\sqrt{\bar{k}_{\theta}}|\phi|^{2}\left(\bar{k}_{\theta}\right)\right]
\end{aligned}
$$

where $A_{i}^{n l}$, which measures the intensity of ion Compton scattering, is given by

$$
A_{i}^{n l}=\frac{4 \pi}{5}\left(\frac{r \hat{s}}{q \rho_{s}}\right)\left(\frac{L_{n}}{\rho_{s}}\right)^{2}\left(\frac{T_{e}}{T_{i}}\right)^{\frac{3}{2}}\left(\frac{L_{s}}{L_{n}}\right)\left(\frac{w}{x_{i}}\right)^{5}\left(\frac{e}{T_{e}}\right)^{2}
$$


also

$$
\begin{aligned}
\frac{\gamma_{e}^{l}}{|\omega|} & =\sqrt{\epsilon}\left(\frac{L_{n}}{L_{s}}\right)^{\frac{1}{2}} \ln \left(\frac{x_{t}}{\Delta}\right) \bar{k}_{\theta} \frac{\bar{\gamma}\left(\bar{k}_{\theta}\right)}{\hat{s}} \\
& \equiv A_{e}^{l} \bar{k}_{\theta} \frac{\bar{\gamma}\left(\bar{k}_{\theta}\right)}{\hat{s}}
\end{aligned}
$$

where $A_{e}^{l}$ and $\bar{\gamma}\left(\bar{k}_{\theta}\right)$ are defined by

$$
\begin{aligned}
& A_{e}^{l}=\sqrt{\epsilon}\left(\frac{L_{n}}{L_{s}}\right)^{\frac{1}{2}} \ln \left(\frac{x_{t}}{\Delta}\right) \\
& \bar{\gamma}\left(\bar{k}_{\theta}\right)=\frac{\vdots}{2 \sqrt{\pi}} I m g_{n},
\end{aligned}
$$

Thus, the saturation condition is given by the following equation,

$$
A_{i}^{n !} \bar{k}_{\theta}^{4} \sqrt{\bar{k}_{\theta}} \frac{\partial}{\partial \bar{k}_{\theta}}\left[\sqrt{\bar{k}_{\theta}}|\phi|^{2}\left(\bar{k}_{\theta}\right)\right]+A_{e}^{l} \bar{k}_{\theta} \frac{\bar{\gamma}\left(\bar{k}_{\theta}\right)}{\hat{s}}=0 .
$$

The solution of the above equation is

$$
|\dot{\phi}|^{2}\left(\bar{k}_{\theta}\right)=\frac{1}{\sqrt{\bar{k}_{\theta}}}|\phi|^{2}(1)-\frac{1}{\sqrt{\bar{k}_{\theta}}} \frac{A_{e}^{l}}{A_{i}^{n^{\prime}}} \int_{1}^{\bar{k}_{\theta}} \bar{k}_{\theta}^{\prime}-\frac{7}{2} \frac{\bar{\gamma}\left(\bar{k}_{\theta}^{\prime}\right)}{\hat{s}} d \bar{k}_{\theta}^{\prime}
$$

In oredr to evaluate the integral in the above equation, a specific instability drive $\bar{\gamma}\left(\bar{k}_{\theta}\right)$ must be utilized. For simplicity, $|\phi|^{2}(1)$ is neglected by setting $\bar{k}_{\theta}=1$ to be the upper wavenumber cutoff of the spectrum, because $|\phi|^{2}\left(\bar{k}_{\theta}\right)$ falls off rapidly for $\bar{k}_{\theta}>1$. As the calculations are straightforward, we just present the results (for $\bar{k}_{\theta}<1$ ) for different collisionality regimes.

\section{Collisionless Regime}

$$
\begin{gathered}
\bar{\gamma}\left(\bar{k}_{\theta}\right) \simeq \bar{\gamma}^{c} \\
\bar{\gamma}^{c}=\epsilon_{n}^{-\frac{3}{2}} e^{-\frac{1}{e_{n}}} \\
|\phi|^{2}\left(\bar{k}_{\theta}\right) \simeq \frac{2}{5} \frac{A_{e}^{l}}{A_{i}^{n l}} \frac{\bar{\gamma}^{c}}{\hat{s}} \bar{k}_{\theta}^{-3} I^{c}\left(\bar{k}_{\theta}\right)
\end{gathered}
$$

with $\epsilon_{n}=\frac{L_{n}}{R}$, and $I^{c}\left(\bar{k}_{\theta}\right)=1-\bar{k}_{\theta}^{\frac{5}{2}}$. 


\section{Dissipative Regime}

$$
\begin{gathered}
\bar{\gamma}\left(\bar{k}_{\theta}\right) \simeq \bar{\gamma}^{d} \bar{k}_{\theta} \\
\bar{\gamma}^{d}=\frac{c_{s}}{\nu_{e f f} L_{n}} \\
|\phi|^{2}\left(\bar{k}_{\theta}\right) \simeq \frac{2}{5} \frac{A_{e}^{l}}{A_{i}^{n l}} \frac{\bar{\gamma}^{d}}{\hat{s}} \bar{k}_{\theta}^{-2} I^{d}\left(\bar{k}_{\theta}\right)
\end{gathered}
$$

where $I^{d}\left(\bar{k}_{\theta}\right)=1-\bar{k}_{\theta}^{\frac{3}{2}}$.

Eqs.(81), (82) predict a power law spectrum, $|\phi|^{2}\left(k_{\theta}\right) \sim k_{\theta}^{-\alpha}$, where $\alpha=2$ and 3 for dissipative and collisionless trapped electron modes, respectively. This spectrum is drawn schematically in Fig.3 which shows the lower cutoff $\bar{k}_{\theta}^{c}$ and upper cutoff 1 . On the same picture, we also show the schematic of the linear growth rate so that one can see that the saturated spectrum is down-shifted towards the lower $k_{\theta}$ region as a consequence of ion Compton scattering. With the spectrum intensity we obtained above, we can calculate the fluctuation level and steady state transport coefficients. Here, the fluctuation level will be calculated, while the calculation of the transport coefficients is left for next section.

The fluctuation level is defined as

$$
\begin{aligned}
& \left\langle\left(\frac{\tilde{n}}{n_{0}}\right)^{2}\right\rangle \simeq\left\langle\left(\frac{e \phi}{T_{e}}\right)^{2}\right\rangle \\
= & \sum_{\substack{m \\
n}}\left|\frac{e}{T_{e}} \phi_{n}\right|^{2} \equiv\left(\frac{e}{T_{e}}\right)^{2} \frac{r}{q} \int \frac{d k_{\theta}}{\Delta} \int_{r-x_{i}}^{r+x_{i}} d r \underset{n}{m_{n}}\left|\phi_{n}^{m}\right|^{2}
\end{aligned}
$$

where the bracket means an average over $\theta$ and $\xi$. By carrying out the $r_{n}$ integration, and noting that $\frac{x_{i}}{\Delta}=\hat{s}\left(k_{\theta} \rho_{s}\right) \frac{L_{s}}{L_{n}} \sqrt{\frac{T_{s}}{T_{i}}}$, we have

$$
\left\langle\left(\frac{e \phi}{T_{e}}\right)^{2}\right\rangle=4\left(\frac{T_{e}}{T_{i}}\right)^{\frac{1}{2}} \frac{L_{s}}{L_{n}}\left(\frac{e}{T_{e}}\right)^{2} \frac{r \hat{s}}{q \rho_{s}} \int_{\bar{k}_{\theta}^{e}}^{1} \bar{k}_{\theta}|\phi|^{2}\left(\bar{k}_{\theta}\right) d \bar{k}_{\theta}
$$

In the above equation, the upper cutoff of the spectrum has been set to be 1 , and the lower cutoff $\bar{k}_{\theta}^{c}$ is determined by the balance between the linear growth rate due to trapped electron excitation and the shear damping rate, i.e.

$$
A_{e}^{l} \bar{k}_{\theta}^{c} \frac{\bar{\gamma}^{(}\left(\bar{k}_{\theta}^{c}\right)}{\hat{s}}=\frac{L_{n}}{L_{s}}
$$


A more accurate determination of $\bar{k}_{\theta}^{c}$ should include the nonlinear transfer rate in the balance equation above which could results in a cutoff smaller than that determined from Eq.(85), i.e. some linearly stable modes will be nonlinearly excited. However, this excitation is very weak, since for small $\bar{k}_{\theta}$ the nonlinear transfer rate is very small. Therefore the difference between the rigorous lower cutoff and $\bar{k}_{\theta}^{c}$ determined from Eq.(85) can be ignored. Now, substituting the steady state spectrum in Eqs.(81),(82) and noting Eqs.(75) and (77), the fluctuation level in various collisionality regimes can be readily calculated. The results are

$$
\left\langle\left(\frac{e \phi}{T_{e}}\right)^{2}\right\rangle \simeq 0.6 \frac{T_{i}}{T_{e}}\left(A_{e}^{l} \frac{\bar{\gamma}}{s}\right) G\left(\bar{k}_{\theta}^{c}\right)\left(\frac{\rho_{s}}{L_{n}}\right)^{2}
$$

In obtaining the above result, the spatial width of the spectrum $w$ has been set equal to $x_{i}$. In the above equation, $\bar{\gamma}$ accounts for the linear growth rate in different collisionality regime and is given by

$$
\bar{\gamma}= \begin{cases}\epsilon_{n}^{-\frac{3}{2}} e^{-\frac{1}{\epsilon_{n}}}, & \text { collisionless regime } \\ \frac{c_{2}}{\nu_{e \rho} L_{n}}, & \text { dissipative regime }\end{cases}
$$

and $G\left(\bar{k}_{\theta}^{c}\right)$ is the function that represents the effects of the spectrum shape and cutoff,

$$
G\left(\bar{k}_{\theta}^{c}\right)= \begin{cases}\frac{1}{\bar{k}_{\theta}^{c}}-\frac{5}{3}+\frac{2}{3} \bar{k}_{\theta}^{c \frac{3}{2}}, & \text { collisionless regime } \\ \ln \frac{1}{k_{\theta}^{c}}-\frac{2}{3}+\frac{2}{3} \bar{k}_{\theta}^{c \frac{3}{2}}, & \text { dissipative regime. }\end{cases}
$$

Since $A_{e}^{l} \propto \sqrt{\frac{L_{n}}{L_{0}}}$, Eq. (86) showes that the fluctuation level predicted is well below the mixing length type estimate of $\frac{e \phi}{T_{a}} \sim \frac{x_{c}}{L_{n}} \sim \sqrt{\frac{L_{e}}{L_{n}}} \frac{\rho_{l}}{L_{n}}$. This is due to the contribution of the radial wavenumbers $k_{r}^{\prime} \sim \mu^{\prime} x_{i}^{\prime} \sim \sqrt{\frac{L_{n}}{L_{n}}} x_{t}^{-1}>x_{t}^{-1}$ to the nonlinear coupling coefficient $\vec{k} \cdot \vec{k}^{\prime} \times \vec{z}$ (since the slab-like eigenmode spectrum extends to $x_{i}$ ), and the weak turbulence reduction factor $\left\langle\frac{y_{a}^{l}}{w_{\bar{k}}}\right\rangle_{\bar{k}} \sim A_{e}^{l} \frac{\bar{q}}{\dot{j}} \ll 1$. Here, $\langle\cdots\rangle_{\bar{k}}$ represents spectrum average.

\section{Turbulent Transport}

Having obtained the wave number spectrum and the fluctuation level, we now consider the effect of trapped electron mode driven turbulence on particle and heat transport. 
The turbulent particle and heat flux are determined by the correlation of the fluctuating radial velocity with the density and pressure fluctuation respectively, i.e.

$$
\begin{aligned}
& \Gamma_{j}=\left\langle\tilde{V}_{r} \tilde{n}_{j}\right\rangle, \\
& Q_{j}=\left\langle\tilde{V}_{r} \tilde{p}_{j}\right\rangle .
\end{aligned}
$$

The bracket represents an ensemble average which in practice can be replaced by an average over the fast variation variables $\theta$ and $\xi$.

For the particle flux, the quasineutrality condition also implies that the particle transport is ambipolar, i.e. the spatial width averaged electron flux should be equal to the ion flux, $\Gamma_{e}=\Gamma_{i}$. In the following, we calculate the electron particle flux directly using the quasi-linear approxmation. This is valid because the trapped electron nonlinear waveparticle interaction is very weak and does not contribute to the saturation criterion. From Eq. (89), the electron particle flux can be rewritten as

$$
\begin{aligned}
& \Gamma_{e}=-\frac{c}{B} \sum_{\substack{m \\
n}} k_{\theta} \operatorname{Im}\left(\tilde{n}_{\substack{m \\
n}}^{e(1)} \tilde{\phi}_{-m}\right) \\
& \equiv-\frac{c}{B} \frac{r}{q} \int \frac{d k_{\theta}}{\Delta} \int_{r-x_{t}}^{r+x_{t}} d r \underset{n}{m} k_{\theta} \operatorname{Im}\left(\tilde{n}_{\substack{m \\
n}}^{e(1)} \tilde{\phi}-m\right)
\end{aligned}
$$

Note in the above equation, the limits for the $r_{n}$ integral have been set to be $r \pm x_{t}$ in order to ensure satisfaction of the well-known relationship between the quasilinear equation and Ime, and preservation of spectrum width averaged ambipolarity. The particle diffusion coefficient is defined as

$$
D=-\frac{\Gamma}{\frac{d N}{d r}}
$$

Substituting Eqs.(10a) and (10b) for $\tilde{n}_{\substack{m \\ m}}^{e(1)}$ in Eq.(91), and making use of the spectrum function $|\phi|^{2}\left(k_{\theta}\right)$ at steady state, we can obtain the particle diffusion coefficient $D$. The result is

$$
D=0.4\left(\frac{T_{i}}{T_{e}}\right)^{\frac{3}{2}}\left(\frac{L_{n}}{L_{s}}\right)^{\frac{1}{2}}\left(A_{e}^{l} \frac{\bar{\gamma}}{\hat{s}}\right)^{2} F\left(\bar{k}_{\theta}^{c}\right) \frac{c_{s} \rho_{s}^{2}}{L_{n}}
$$


where the function $F\left(\bar{k}_{\theta}^{c}\right)$ is given by

$$
F\left(\bar{k}_{\theta}^{c}\right)= \begin{cases}\frac{5}{7}-\bar{k}_{\theta}^{c}+\frac{2}{7} \bar{k}_{\theta}^{c \frac{7}{2}}, & \text { collisionless regime } \\ \frac{1}{15}-\frac{1}{5} \bar{k}_{\theta}^{c 3}+\frac{2}{15} \bar{k}_{\theta}^{c \frac{9}{2}}, & \text { dissipative regime. }\end{cases}
$$

and the instability source term $\bar{\gamma}$ is given by Eq. (87).

Now let's turn to the discussion of the heat transport. From Eq.(90), the heat flux can be rewritten as,

$$
Q^{j}=-\frac{c}{B} \sum_{\substack{m \\ n}} k_{\theta} \operatorname{Im}\left[\left(\tilde{p}_{n}^{j(1)}+\underset{n}{\tilde{p}_{m}^{j(3)}}+\cdots\right) \tilde{\phi}-m\right]
$$

where $\tilde{p}_{n}^{j(l)}$ is the lth order pressure response in the weak turbulence expension. The first term in the above equation corresponds to the quasilinear heat flux. The second term corresponds to the heat flux caused by nonlinear wave-particle interaction. For electrons, the quasilinear flux is dominant, since nonlinear trapped electron-wave interaction is weak. For ions, the ion Compton scattering induced heat flux is dominant, since the quasilinear flux caused by the shear damping is negligible. As a consequence, we can write the thermal flux for ions and electrons as:

$$
\begin{aligned}
& Q^{i}=-\frac{c}{B} \sum_{\substack{m \\
n}} k_{\theta} \operatorname{Im}\left(\tilde{p}_{n}^{i(3)} \tilde{\phi}_{-m}\right) \\
& Q^{e}=-\frac{c}{B} \sum_{\substack{m \\
n}} k_{\theta} \operatorname{Im}\left(\tilde{p}_{n}^{e(1)} \tilde{\phi}_{-m}\right)
\end{aligned}
$$

where $\tilde{p}_{n}^{i(3)}$ and $\tilde{p}_{n}^{e(1)}$ are given by

$$
\begin{aligned}
& \tilde{p}_{m}^{i(3)}=\int d^{3} \vec{v} J_{0}\left(\frac{k_{\perp} v_{\perp}}{\Omega_{i}}\right) \frac{1}{2} m_{i} v^{2} h_{\frac{m}{n}}^{i(3)} \\
& \tilde{p}_{m}^{e(1)}=\int_{-\pi}^{\pi} \frac{d \theta}{2 \pi} e^{i(n q-m) \theta} \int_{t} d^{3} \vec{v} \frac{1}{2} m_{e} v^{2} \hat{h}_{n}^{e(1)}
\end{aligned}
$$

The thermal flux defined as above can be divided into two terms,

$$
Q^{j}=Q_{c v}^{j}+Q_{c d}^{j}
$$


On the right side of the above equation, the first term $Q_{o v}^{j}$ is called convected thermal flux, and is driven by the density gradient. Since this term results from the transport of heat by particle diffusion, we may write this term as $Q_{c v}^{j}=T_{j} \Gamma$. The second term $Q_{c d}^{j}$ is called the conducted thermal flux. It is driven by the temperature gradient. We define the heat transport coefficient $\chi_{j}$ by

$$
\chi_{j}=-\frac{Q_{c d}^{j}}{N \frac{d T_{j}}{d r}}
$$

Note that in order to calculate the thermal transport coefficients, finite temperature gradients are required. The transport coefficients determined in this way usually are nonlinear functions of the temperature gradient since the linear growth rate $\gamma_{e}^{l}$ also depends on the $\nabla T_{e}$. For self-consistency, strictly speaking, we must require that the temperature gradients we introduce to calculate the thermal transport coefficients be relatively weak so that our previous assumptions are not violated. In such cases, the transport coefficients do not depend strongly on the temperature gradient. Now substituting $\underset{n}{h_{m}^{i(3)}}$ from Eq.(34a) and $\hat{h}_{n}^{e(1)}$ from Eq.(8b) into the Eqs.(95b) and (96b) and carrying out the velocity integrals, we can obtain the third order ion pressure response and the first order electron pressure response. Substituting these responses into Eqs.(95a) and (95b), and noting the definition for $\chi_{j}$ in Eq.(97), we obtain

$$
\begin{aligned}
\chi^{i} & =0.8\left(\frac{T_{i}}{T_{e}}\right)^{\frac{3}{2}}\left(\frac{L_{n}}{L_{s}}\right)^{\frac{1}{2}}\left(A_{e}^{l} \frac{\bar{\gamma}}{\hat{s}}\right)^{2} F\left(\bar{k}_{\theta}^{c}\right) \frac{\rho_{s}^{2} c_{s}}{L_{n}} \\
\chi^{e} & =0.8\left(\frac{T_{i}}{T_{e}}\right)^{\frac{3}{2}}\left(\frac{L_{n}}{L_{s}}\right)^{\frac{1}{2}}\left(A_{e}^{l} \frac{\bar{\gamma}}{\hat{s}}\right)^{2} H\left(\bar{k}_{\theta}^{c}\right) \frac{c_{s} \rho_{s}^{2}}{L_{n}}
\end{aligned}
$$

where $H\left(\bar{k}_{\theta}^{c}\right)$ is given by

$$
H\left(\bar{k}_{\theta}^{c}\right)= \begin{cases}\frac{1}{\epsilon_{n}}\left[\left(\frac{1}{\epsilon}-\frac{3}{2}\right) G\left(\bar{k}_{\theta}^{c}\right)-\frac{3}{2} F\left(\bar{k}_{\theta}^{c}\right)\right], & \text { for collisionless mode } \\ \frac{1}{\pi}\left[25 F\left(\bar{k}_{\theta}^{c}\right)+9 \bar{k}_{\theta}^{c} G\left(\bar{k}_{\theta}^{c}\right)\right], & \text { for dissipative mode. }\end{cases}
$$

For trapped electron driven drift wave turbulence, the momentum diffusivity $\chi_{\phi} \simeq \chi_{i}$. The particle transport coefficient, the heat transport coefficients shown in Eqs.(93), (98), (99) are substantially smaller than the mixing length type estimate. This is due to the effect 
that trapped electron response is radially localized to $x \leq \Delta<x_{t}$, the weak turbulence reduction factor $\left\langle\frac{r_{a}^{\prime}}{w_{\vec{k}}}\right\rangle_{\vec{k}}^{2}$, and the reduction in the fluctuation level which results from the enhanced ion Compton scattering.

Now, let's discuss the practical implications of Eq.(93), (98) and (99) for tokamak transport. First, we notice that $L_{s}=\hat{s}^{-1} q R$ and $A_{e}^{l} \propto\left(\frac{L_{a}}{L_{0}}\right)^{\frac{1}{2}}$. Hence,

$$
D, \chi_{e}, \chi_{i} \propto\left(\frac{\hat{s}}{q} \frac{L_{n}}{R}\right)^{\frac{3}{2}}
$$

i.e. all the transport coefficients have favorable major radius dependence. This favorable major radius dependence results from the radial localization of trapped electron response, and therefore will occur in all collisionality regimes. Second, in the dissipative regime, $\bar{\gamma}=\frac{c_{.}}{L_{n} \nu_{e \rho}} \propto T_{e}^{2} m_{i}^{-\frac{1}{2}} n^{-1}, c_{s} \rho_{s} \propto m_{i}^{\frac{1}{2}} T_{e}^{\frac{3}{2}}$, we have,

$$
D \sim \chi_{e} \sim \chi_{i} \propto T_{e}^{\frac{11}{2}} n^{-2} m_{i}^{-\frac{1}{2}}
$$

i.e. all the transport coefficients in the dissipative regime have a favorable dependence on both the density and the ion mass. These favorable dependences do not occur in the collisionless regime. Third, we observe the manifestation of confinement irnprovement in peaked profile, collisionless regimes. This is apparent from the dependence of the transport coefficients $\operatorname{cn} \exp \left(-\frac{R}{L_{n}}\right)$, which is furdamental to the collisionless trapped electron growth rate.

\section{Discussion and Conclusion}

In this paper, a kinetic theory of trapped electron driven drift wave turbulence which treats electron and ion nonlinearity on an equal footing is presented. The principal results of this investigation are summarized in Table I. In particular, we find:

i.) the disparity between the basic scales $\Delta<x_{t}<x_{i}$ indicates that ion Compton scattering is the dominant nonlinear process for slab-like trapped electron driven drift wave turbulence. 
ii.) ion Compton scattering induces spectral transfer to long wavelengths. The resulting spectrum $|\dot{\phi}|^{2}\left(k_{\theta}\right) \sim k_{\theta}^{-\alpha}$ ( $\alpha=2$ and 3 for dissipative and collisionless regimes, respectively) is heavily damped for $k_{\theta} \rho_{s}>1$ and cutoff by shear damping at $\bar{k}_{\theta}^{c}$.

iii.) saturated fluctuation levels are smaller than those predicted by naive mixing length theory $\frac{e \phi}{T_{a}} \sim \frac{x_{s}}{L_{n}} \sim \sqrt{\frac{L_{n}}{L_{n}}} \frac{\rho_{e}}{L_{n}}$. This disparity is due to the appearence of $k_{r}^{\prime} \rho_{i} \sim$ 1 contributions to the coupling coefficient $\vec{k} \cdot \vec{k}^{\prime} \times \vec{e}_{\|}$and to the effects of "weak turbulence" factors $\left\langle\frac{\gamma_{c}^{l}}{w_{\bar{k}}}\right\rangle_{\vec{k}} \sim A_{e}^{l} \frac{\bar{\gamma}}{\dot{j}} \ll 1$.

iv.) the transport coefficients $\chi_{e}, \chi_{i}$, and $D$ have been determined using the calculated fluctuation spectrum. The results manifest explicit dependence on the spectral shape and cutoffs, and are smaller than the mixing length estimate predictions due to effects of localization of the trapped electron response to $\Delta\left(<x_{t}<x_{i}\right)$, the "weak turbulence" factor $\left(A_{e}^{l} \frac{\bar{\gamma}}{\dot{j}}\right)^{2}$ and the reduction in fluctuation levels discussed above.

These results have a number of interesting implications for tokamak confinement theory, which include:

i.) the appearence of the robust favorable major radius scaling, due to the trapped electron layer localization effect.

ii.) the manifestation of favorable isotope scaling in the dissipative trapped electron regime. This favorable isotope scaling is accompanied by a transition from $\chi \sim 1 / n$ to $\chi \sim 1 / n^{2}$ scaling.

iii.) the manifestation of confinement improvement in the peaked profile, collisionless trapped electron regime. This trend is a direct consequence of the $\exp \left(-\frac{R}{L_{n}}\right)$ dependence of the collisionless trapped electron mode growth rate.

iv.) finally (and most important!), we observed that short wavelength drift wave turbulence levels and transport coefficients are considerably smaller than predictions derived from naive mixing length estimates. This result suggests that longer wavelength toroidal drift waves and trapped ion modes are probably the dominant agents for electron driven core transport in tokamaks. 
At this point, it is appropriate to briefly comment on the implications of this work for longer wavelength, toroidicity-induced trapped electron mode turbulence. In contrast to the slab-like branch, toroidal modes are standing waves, with coupled poloidal subbarmonics of width $\Delta x \leq \frac{1}{k_{\theta} \dot{j}}$ and $k_{\|} v_{i} \ll \omega$. It is apparent from the analysis presented in this paper that: (a) ion Compton scattering is ineffectual for toroidal modes, since $\Delta\left(k_{\|} v_{i}\right) \ll \omega$. (b) the role of electron-mediated and ion-mediated nonlinearity are comparable, since $\Delta x \sim \Delta$. (c) it is likely that $\vec{E} \times \vec{B}$ mode coupling is the dominant ion-mediated nonlinear process, since $k_{\perp}^{2} \rho_{s}^{2} \ll 1$ for toroidal drift wave ${ }^{12}$. In contrast to the "conventional wisdom", it is easily seen that such $\vec{E} \times \vec{B}$ mode coupling transfers energy to small scales, even in quasi-two dimensional systems. ${ }^{16}$ In combination with the trapped electron mediated transfer to large $k_{\theta}$ discussed in this paper, such ion mode coupling suggests the energy flow in long wavelength drift wave turbulence is toward small scales. A detailed theoretical analysis of toroidicity induced drift wave turbulence is underway and will be reported in a future publication.

We want to remark that in our calculation the electron temperature gradient effect has been neglected. Finite electron temperature gradient can enhance the linear growth rate $^{\perp}$, but will not change the trapped electron dynamics. Since the linear tineory will change when finite $\eta_{e}$ is included, the steady state spectra can be expected to change, as well. However, our results obtained in this paper can be easily modified to include this change.

Finally, the results presented here further underscore the need for gyrokinetic ${ }^{17}$ and bounce-kinetic ${ }^{15}$ simulations of trapped electron mode turbulence. Similarly, coupled fluctuation and transport studies of low frequency turbulence, particularly in flexible magnetic configurations such as stellarators, ${ }^{18}$ should be vigorously pursued.

\section{Acknowledgments}

This work was supported by the U. S. Department of Energy under Grant No. DEFG05-5038s with the University of Texas at Austin and Grant No. DE-FG03-50388-53275 
with the University of California, San Diego. P. H. Diamond would like to acknowledge support from an Alfred P. Sloan Research Fellowship and a National Science Foundation Presidential Young Investigator Award. 


\section{References}

1. W. M. Tang, Nuclear Fusion 18, 1089 (1978).

2. P. J. Catto, and K. T. Tsang, Phys. Fluids 21, 1381 (1978).

3. W. M. Manheimer, K. R. Chu, E. Ott, and J. P. Boris, Phys. Rev. Lett. 37, 286 (1976).

4. A. Rogister, and G. Hasselberg, Phys. Fluids 26, 1467 (1983).

5. P. L. Similon, and P. H. Diamond, Phys. Fluids 27, 916 (1984).

6. L. Chen, R. L. Berger, J. G. Lominadze, M. N. Rosenbluth, and P. H. Rutherford, Phys. Rev. Lett. 39, 754 (1977).

7. P. H. Diamond, P. L. Similon, P. W. Terry, C. W. Horton, S. M. Mahajan, J. D, Meiss, MI. N. Rosenbluth, K. Swartz, T. Tajima, R. D. Hazeltine, D. W. Ross, in Plasma Physics and Controlled Nuclear Fusion Research (IAEA, Vienna, 1982), Vol.I, p.259.

8. R. Waltz, Phys. Fluids 28, 577 (1985).

9. A. Hasegawa, and K. Mima, Phys. Fluids 21, 87 (1978).

10. T. Tange, K. Nishikawa, and A. K. Sen, Phys. Fluids 25, 1592 (1982).

11. L. D. Pearlstein, and H. L. Berk, Phys. Rev. Lett. 23, 220 (1969).

12. L. Chen, and C. Z. Cheng, Phys. Fluids 23, 2242 (1980).

13. R. Z. Sagdeev and A. A. Galeev, in Nonlinear Plasma Theory, edited by T. M. O'Neil and D. L. Book (Benjamin, New York, 1969), p.89.

14. E. A. Frieman, and L. Chen, Phys. Fluids 25, 502 (1982).

15. F. Y. Gang, and P. H. Diamond, submitted to Phys. Fluids B May, 1990.

16. P. H. Diamond, and H. Biglari, submitted to Phys. Rev. Lett. June, 1990.

17. W. W. Lee, Phys. Fluids 26, 556 (1983).

18. B. A. Carreras, N. N. Dominguez, P. H. Diamond, and L. Garcia, Bull. Am. Phys. Soc. 34. 2046 (1989). 
Table I. Summary of Principal Results

\begin{tabular}{|c|c|c|}
\hline Quantites & $\begin{array}{r}\text { Collisionless Regime } \\
\qquad\left(\omega>\omega_{d e}>v_{e f f}\right)\end{array}$ & $\begin{array}{r}\text { Dissipative Regime } \\
\left(\nu_{\text {eff }}>\omega>\omega_{d c}\right)\end{array}$ \\
\hline $\bar{\gamma}$ & $\epsilon_{n}^{-\frac{3}{2}} e^{-\frac{1}{\epsilon_{n}}}$ & $\frac{c_{1}}{\nu_{e f f} L_{n}}$ \\
\hline$|\phi|^{2}\left(\bar{k}_{\theta}\right)$ & $0.4 \frac{A_{t}^{l}}{A_{i}^{t}} \frac{\bar{q}}{3} \bar{k}_{\theta}^{-3} I\left(\bar{k}_{\theta}\right)$ & $0.4 \frac{A_{n}^{l}}{A_{i}^{t}} \frac{\bar{q}}{s} \bar{k}_{\theta}^{-4} I\left(\bar{k}_{\theta}\right)$ \\
\hline$\left(\frac{\tilde{h}}{n_{0}}\right)^{2}$ & $0.6 \frac{T_{i}}{T_{e}}\left(A_{e}^{l} \frac{\bar{\gamma}_{j}}{j}\right) G\left(\bar{k}_{\vartheta}^{c}\right)\left(\frac{\rho_{e}}{L_{n}}\right)^{2}$ & same \\
\hline$D$ & $0.4\left(\frac{T_{i}}{T_{\theta}}\right)^{\frac{3}{2}}\left(\frac{L_{n}}{L_{0}}\right)^{\frac{1}{2}}\left(A_{e}^{l} \frac{\bar{\gamma}}{\dot{j}}\right)^{2} F\left(\bar{k}_{\theta}^{c}\right) \frac{c_{0} \rho_{t}^{2}}{L_{n}}$ & same \\
\hline$\chi_{i}$ & $0.8\left(\frac{T_{i}}{T_{e}}\right)^{\frac{3}{2}}\left(\frac{L_{n}}{L_{0}}\right)^{\frac{1}{2}}\left(A_{e}^{l} \frac{\hat{\gamma}}{\dot{j}}\right)^{2} F\left(\bar{k}_{\theta}^{c}\right) \frac{c_{0} \rho_{1}^{2}}{L_{n}}$ & same \\
\hline$\chi_{e}$ & $0.8\left(\frac{T_{i}}{T_{e}}\right)^{\frac{3}{2}}\left(\frac{L_{n}}{L_{e}}\right)^{\frac{1}{2}}\left(A_{e}^{l} \bar{\gamma} \frac{\bar{j}}{\bar{j}}\right)^{2} H\left(\bar{k}_{\theta}^{c}\right) \frac{c_{0} \rho_{1}^{2}}{L_{n}}$ & same \\
\hline$I\left(\bar{k}_{\theta}\right)$ & $1-\bar{k}_{\theta}^{\frac{3}{2}}$ & $1-\bar{k}_{\theta}^{\frac{3}{2}}$ \\
\hline$G\left(\bar{k}_{\theta}^{c}\right)$ & $\frac{1}{k_{g}^{c}}-\frac{5}{3}+\frac{2}{3} \bar{k}_{\theta}^{c \frac{3}{2}}$ & $\ln \frac{1}{k_{\theta}^{c}}-\frac{2}{3}+\frac{2}{3} \bar{k}_{\theta}^{c \frac{3}{2}}$ \\
\hline$F\left(\bar{k}_{\theta}^{c}\right)$ & $\frac{5}{7}-\bar{k}_{\theta}^{c}+\frac{2}{7} \bar{k}_{\theta}^{c \frac{7}{2}}$ & $\frac{1}{15}-\frac{1}{3} \bar{k}_{\theta}^{c^{3}}+\frac{2}{15} \bar{k}_{\theta}^{\frac{2}{2}}$ \\
\hline$H\left(\bar{k}_{\theta}^{c}\right)$ & $\frac{1}{\epsilon_{n}}\left[\left(\frac{1}{\epsilon_{n}}-\frac{3}{2}\right) G\left(\bar{k}_{\theta}^{c}\right)-\frac{3}{2} F\left(\bar{k}_{\theta}^{c}\right)\right]$ & $\frac{1}{\pi}\left[25 F\left(\bar{k}_{\theta}^{c}\right)+9 \bar{k}_{\theta}^{c} G\left(\bar{k}_{\theta}^{c}\right)\right]$ \\
\hline $\bar{k}_{\theta}^{c}$ & $\frac{L_{n}}{L_{0}} \frac{j}{A_{e}^{!} \bar{\gamma}} \propto\left(\frac{L_{n}}{L_{0}}\right)^{\frac{1}{2}}$ & $\left(\frac{L_{n}}{L_{0}} \frac{\dot{g}}{A_{a}^{l} \bar{\gamma}}\right)^{\frac{1}{2}} \propto\left(\frac{L_{n}}{L_{0}}\right)^{\frac{1}{4}}$ \\
\hline
\end{tabular}




\section{Figure Captions}

Figure 1: This figure depicits the linear eigenmode structure of a slab-like drift wave (Pearlstein-Berk outgoing wave) with three different spatial scale lengths $\Delta, x_{t}$, and $x_{i}$. Two neigbouring mode rational surface of $\left(\begin{array}{c}m \\ n\end{array}\right)$ and $\left(\begin{array}{c}m+1 \\ n\end{array}\right)$ are also shown on the diagram.

Figure 2: This figure depicits the linear dispersion relation for slab-like drift mode. On the diagram, two distant wavenumbers $k_{\theta}$ and $1 / k_{\theta} \rho_{s}^{2}$, which conrrespond to the same frequency $\omega\left(k_{\theta}\right)$, are shown.

Figure 3: This figure depicits the shape and cutoffs of the saturated spectrum $|\phi|^{2}\left(k_{\theta}\right)$. $\bar{k}_{\theta}^{c}$ is the lower cutoff determined by the balance between the linear growth rate and the shear damping rate. On the same diagram, the schematic curve of the linear growth rate is also shown. 
END

DATE FILMED

$1 2 \longdiv { 1 1 1 9 0 }$ 


\section{0}

